\title{
Molecular gas in NUclei of GAlaxies (NUGA)
}

\section{A complete gravity torque map of NGC 4579: new clues to bar evolution}

\author{
S. García-Burillo ${ }^{1}$, S. Fernández-García ${ }^{1}$, F. Combes ${ }^{2}$, L. K. Hunt ${ }^{3}$, S. Haan ${ }^{4}$, E. Schinnerer ${ }^{4}$, F. Boone ${ }^{2}$, \\ M. Krips ${ }^{5}$, and I. Márquez ${ }^{6}$ \\ 1 Observatorio Astronómico Nacional (OAN)-Observatorio de Madrid, Alfonso XII 3, 28014 Madrid, Spain \\ e-mail: [s.gburillo;s.fernandez]@oan.es \\ 2 Observatoire de Paris, LERMA, 61 Av. de l'Observatoire, 75014 Paris, France \\ e-mail: [francoise. combes; frederic. boone] @obspm.fr \\ 3 Istituto di Radioastronomia/CNR, Sez. Firenze, Largo Enrico Fermi 5, 50125 Firenze, Italy \\ e-mail: hunt@arcetri.astro.it \\ ${ }^{4}$ Max-Planck-Institut für Astronomie, Königstuhl 17, 69117 Heidelberg, Germany \\ e-mail: haan@mpia.de; schinner@mpia-hd.mpg.de \\ 5 Center for Astrophysics, SMA project, 60 Garden Street, MS 78 Cambridge, MA-02138, USA \\ e-mail:mkrips@cfa.harvard.edu \\ 6 Instituto de Astrofísica de Andalucía (CSIC), Apdo 3004, 18080 Granada, Spain \\ e-mail: isabel@iaa.es
}

Received 18 July 2008 / Accepted 14 January 2009

\section{ABSTRACT}

\begin{abstract}
In this paper we create a complete gravity torque map of the disk of the LINER/Seyfert 1.9 galaxy NGC 4579 . We quantify the efficiency of angular momentum transport and search for signatures of secular evolution in the fueling process from $r \sim 15 \mathrm{kpc}$ down to the inner $r \sim 50 \mathrm{pc}$ around the active galactic nucleus (AGN). We use both the $1-0$ and $2-1$ line maps of ${ }^{12} \mathrm{CO}$ obtained with the Plateau de Bure Interferometer (PdBI) as part of the NUclei of Galaxies-(NUGA)-project. The CO(1-0) and CO(2-1) PdBI maps at $2.0^{\prime \prime} \times 1.3^{\prime \prime}$ and $1.0^{\prime \prime} \times 0.6^{\prime \prime}$ resolution, respectively, include the short spacing correction provided by IRAM-30 m data. We derive the stellar potential from an NIR ( $K$ band) wide field image of the galaxy. The $K$-band image, which reveals a large-scale stellar bar and a weak nuclear oval, together with a high-resolution Hi map of NGC 4579 obtained with the Very Large Array (VLA), allow us to extend the gravity torque analysis to the outer $r \geq 2 \mathrm{kpc}$ disk.

The bulk of the gas response traced by the new CO PdBI+30 m maps of NGC 4579 presented here follows the expected gas flow pattern induced by the bar potential in the presence of two inner Lindblad resonances (ILR) at $r \sim 500 \mathrm{pc}$ and $r \sim 1.3 \mathrm{kpc}$. The morphology of the outer disk traced by Hi suggests that the neutral gas is currently piling up in a pseudo-ring formed by two winding spiral arms that are morphologically decoupled from the bar structure. The pseudo-ring is located inside the bar corotation $\left(r_{\mathrm{CR}} \sim\right.$ $6 \mathrm{kpc}$ ) and close to the predicted position of the ultra harmonic resonance (UHR) $\left(r_{\mathrm{UHR}} \sim 3.8 \mathrm{kpc}\right)$. The derived gravity torque budget in NGC 4579 shows that inward gas flow is occurring on different spatial scales in the disk. In the outer disk, the decoupling of the spiral allows the gas to efficiently populate the UHR region, and thus produce net gas inflow on intermediate scales. The corotation barrier seems to be overcome by secular evolution processes. The gas in the inner disk $(r \leq 2 \mathrm{kpc})$ is efficiently funneled by gravity torques down to $r \sim 300 \mathrm{pc}$. Closer to the AGN ( $r<200 \mathrm{pc}$ ), gas feels negative torques due to the combined action of the large-scale bar and the inner oval. The two $m=2$ modes act in concert to produce net gas inflow down to $r \sim 50 \mathrm{pc}$, providing clear smoking gun evidence of inward gas transport on short dynamical timescales ( $\sim 1-3$ rotation periods).
\end{abstract}

Key words. galaxies: individual: NGC 4579 - galaxies: ISM - galaxies: kinematics and dynamics - galaxies: nuclei galaxies: Seyfert - radio lines: galaxies

\section{Introduction}

Nuclear activity in galaxies is understood as the result of the feeding of supermassive black holes (SMBH), which are suspected to be a common component in most, perhaps all, galaxies with a significantly massive bulge (e.g., see review by Ferrarese $\&$ Ford 2005, and references therein). Active galactic nuclei (AGN) must be fed with material coming from the disk of the host galaxy. The gas supply, lying originally far away from the gravitational influence of the black hole, must lose virtually all

* Based on observations carried out with the IRAM Plateau de Bure Interferometer. IRAM is supported by INSU/CNRS (France), MPG (Germany) and IGN (Spain). of its angular momentum in the fueling process. While for highluminosity AGNs, a good correlation between the presence of $\sim \mathrm{kpc}$-scale non-axisymmetric perturbations and the existence of activity has been found (e.g., Hutchings \& Neff 1992), a similar correlation in low-luminosity AGNs (LLAGNs) is weak at most (e.g., Mulchaey \& Regan 1997; Knapen et al. 2000; Márquez et al. 2000; Combes 2003). The search for a universal mechanism for AGN feeding in LLAGNs is probably complicated by the brevity of the AGN duty cycle $\left(\sim 10^{6-7}\right.$ years), which might be shorter than the lifetime of the feeding mechanism itself (e.g., Wada 2004; Hopkins \& Hernquist 2006; King \& Pringle 2007). In spite of many theoretical and observational efforts, a solution to the LLAGN fueling problem has thus far remained elusive (e.g., see review by Martini 2004). 
The study of the content, distribution and kinematics of interstellar gas is a key to understanding the origin and maintenance of nuclear activity in galaxies. The processes involved in AGN fueling encompass a wide range of scales, both spatial and temporal, which have to be studied. Probing the gas flow from the outer disk down to the central engine of an AGN host requires the use of specific tracers of the interstellar medium adapted to follow the change of phase of the gas as a function of radius. As most of the neutral gas in galactic nuclei is in the molecular phase, low- $J$ rotational transitions of carbon monoxide $(\mathrm{CO})$ are the best choice to undertake high spatial resolution $\left(\simeq 1^{\prime \prime}\right)$ interferometer mapping of the central kiloparsec disks of AGNs. On the other hand, the $21 \mathrm{~cm}$ line emission of atomic hydrogen (HI) is best suited to tracing the distribution and kinematics of neutral gas in the outer disk reservoirs of AGNs ( $r \geq 5 \mathrm{kpc}$ ) with moderate spatial resolution $\left(\simeq 10-20^{\prime \prime}\right)$ using an interferometer.

The NUclei of GAlaxies-NUGA-project, described by García-Burillo et al. (2003a,b), is the first high-resolution $\left(\sim 0.5^{\prime \prime}-1^{\prime \prime}\right){ }^{12} \mathrm{CO}$ survey of 12 nearby $(D=4-40 \mathrm{Mpc})$ LLAGNs including the full sequence of activity types (Seyferts, LINERs and transition objects). Observations, carried out with the IRAM Plateau de Bure Interferometer (PdBI), have been completed as of early 2004. NUGA surpasses in both spatial resolution $(10-100 \mathrm{pc})$ and sensitivity $(3 \sigma$-detection limit $\simeq$ a few $10^{5-6} M_{\odot}$ ) the previous surveys of nearby AGN conducted at the Owens Valley Radio Observatory-OVRO (Baker 2000; Jogee et al. 2001) and at the Nobeyama Radio Observatory-NRO (Kohno et al. 2001). NUGA maps allow us to probe the gas flows at critical spatial scales $(<100 \mathrm{pc})$ where secondary modes embedded in the kpc-scale perturbations are expected to take over in the fueling process. A bottom line result of NUGA is the identification of a wide range of morphologies in the central kpc-disks of these LLAGNs. This includes one-arm spirals or $m=1$ instabilities (NGC 4826: García-Burillo et al. 2003b; NGC 3718: Krips et al. 2005), symmetric rings (NGC 7217: Combes et al. 2004; NGC 3147: Casasola et al. 2008), as well as gas bars and two-arm spirals (NGC 4569: Boone et al. 2007; NGC 2782: Hunt et al. 2008; NGC 6574: Lindt-Krieg et al. 2008). This result suggests an evolutionary scenario where several mechanisms cooperate to feed the central engines of LLAGNs. More recently, we have completed a parallel HI survey of NUGA galaxies with the Very Large Array (VLA). The aim of this extension of the NUGA project is to use the HI emission as a probe of the distribution and kinematics of neutral gas in the outer disk regions of the galaxies (Haan et al. 2008a, 2008b). The overall gas response to the stellar potential throughout the disk is characterized with the help of high-resolution optical and NIR images of the galaxies.

In a previous paper, García-Burillo et al. (2005, hereafter GB05) developed a method to study the efficiency of stellar gravity torques for the removal of gas angular momentum. In the four galaxies studied there, including NGC 4579, the subject of this paper, paradoxically no evidence of gas inflow was found. This method has been subsequently applied to two more NUGA galaxies (NGC 2782 and NGC 3147), but only in NGC 2782 did we find systematically negative torques down to the resolution limit of the images (Hunt et al. 2008; Casasola et al. 2008), certainly fueling the nuclear starburst, and perhaps later fueling the AGN. Indeed, finding "smoking gun" evidence of AGN fueling is proving to be quite challenging, perhaps because of the short-lived nature of the mechanisms responsible.

In this paper, we reanalyze the torque budget in NGC 4579 by including new $\mathrm{CO}(1-0)$ maps, HI maps, single-dish $\mathrm{CO}$ observations, and a new $K$-band image from which we derive the gravitational potential; the new evidence seems to suggest that NGC 4579 is the best NUGA case so far for gas inflow.

\subsection{The LINER/Seyfert 1.9 NGC 4579}

NGC 4579 is a SAB(rs)b galaxy classified as an intermediate type 1 object (LINER/Seyfert 1.9) by Ho et al. (1997). The AGN nature of NGC 4579 is confirmed by the detection of broad $\mathrm{H} \alpha$ and UV lines (Stauffer 1982; Filippenko \& Sargent 1985; Barth et al. 1996, 2001; Maoz et al. 1998). It also has an unresolved nuclear hard X-ray (variable) source with a prominent broad Fe K $\alpha$ line (Terashima et al. 2000; Ho et al. 2001; Eracleous et al. 2002; Dewangan et al. 2004). A non-thermal radio continuum source is detected at the position of the AGN (Hummel et al. 1987; Ho \& Ulvestad 2001; Ulvestad \& Ho 2001; Krips et al. 2007). Hubble Space Telescope (HST) optical and UV images resolve the central kiloparsec disk of NGC 4579 and show a nuclear component surrounded by a highly structured disk (Pogge et al. 2000; Contini 2004).

GB05 used the PdBI CO(2-1) map of NGC 4579 to study the distribution and kinematics of molecular gas in the inner $r \leq$ $1 \mathrm{kpc}$ disk of NGC 4579. Only $\mathrm{CO}(2-1)$ data were used in this analysis, and the short spacing correction was not considered. Most of the molecular gas in the central kiloparsec was seen to be concentrated in a two arm spiral structure traceable from $r \sim$ $1 \mathrm{kpc}$ down to $r \sim 200 \mathrm{pc}$.

The gravity torque map discussed by GB05 was derived from an $I$-band HST image of NGC 4579 covering the central $r \leq$ $1.5 \mathrm{kpc}$ disk. The inferred overall mass inflow budget was seen to be clearly negative down to $r \sim 200 \mathrm{pc}$ due to the action of the large-scale bar of NGC 4579 (Fig. 11 of GB05). However, GB05 concluded that inside $r \sim 200$ pc stellar torques are positive and, consequently, they do not help AGN feeding at present. The gas directly responsible for the Seyfert activity in NGC 4579 could have been brought to the center during a previous fueling phase, while the bar and the strong $m=1$ mode, identified in the stellar potential derived from the $I$-band image of the galaxy, would be emptying the region inside $r \sim 200 \mathrm{pc}$. According to this study, AGN fueling is presently quenched in NGC 4579.

\subsection{Aims of this paper}

In this paper we re-determine the gravitational torque budget in the disk of NGC 4579, using a set of new data for the gas and the stellar distribution. The new torque map in the inner disk is derived using both the $1-0$ and $2-1$ line maps of ${ }^{12} \mathrm{CO}$ obtained within the NUGA project. The maps used in this work include now the short spacing correction provided by the new IRAM-30 m data obtained in the two lowest-order transitions of $\mathrm{CO}$, and thus give a more reliable estimate of the implied gas flow timescales, compared to the first estimate of GB05. Although the missing zero-spacing flux is expected not to have a dramatic influence on the value of gravity torques in the circumnuclear disks of galaxies (see discussion in Sect. 2.1 of GB05), it is worthwhile to test this hypothesis. Furthermore, we have obtained a $K$-band wide-field image of the galaxy from which we derive the stellar potential. This allows us to minimize the effects of extinction that were present in the HST I-band image used in our first gravity torque analysis (GB05). Indeed, as we shall show, the $m=1$ mode in the HST $I$-band image of NGC 4579 was spurious, resulting from dust extinction. Finally, the large field-of-view of the $K$-band image, together with the availability of a new high-resolution and sensitivity HI map of NGC 4579 
Table 1. Observational parameters of NGC 4579.

\begin{tabular}{lll}
\hline \hline Parameter & Value & Reference \\
\hline$\alpha_{J 2000}^{a}$ & $12^{\mathrm{h}} 37^{\mathrm{m}} 43.52^{\mathrm{s}}$ & This work \\
$\delta_{J 2000}^{a}$ & $11^{\circ} 49^{\prime} 05.5^{\prime \prime}$ & This work \\
$V_{\text {hel }}$ & $1466 \mathrm{~km} \mathrm{~s}^{-1}$ & This work \\
RC3 type & SAB $(\mathrm{rs}) \mathrm{b}$ & NED \\
AGN type & LINER/Sy1.9 & NED \\
t type & 2.8 & LEDA \\
Inclination & $36^{\circ}$ & GB05, this work \\
Position Angle & $95^{\circ}$ & GB05, this work \\
Distance & $19.8 \mathrm{Mpc}\left(1^{\prime \prime}=97 \mathrm{pc}\right)$ & GB05 \\
$M_{\mathrm{B}}$ & $-21.68 \mathrm{mag}$ & LEDA \\
$M(\mathrm{HI})$ & $1.7 \times 10^{8} M_{\odot}$ & Haan et al. (2008a) \\
$M\left(\mathrm{H}_{2}\right)$ & $5 \times 10^{8} M_{\odot}$ & This work \\
$L_{\mathrm{FIR}}$ & $9.6 \times 10^{9} L_{\odot}$ & Sanders et al. (2003) \\
\hline
\end{tabular}

${ }^{a}$ Dynamical center.

(Haan et al. 2008a), allow us to extend the gravity torque analysis to the outer disk of the galaxy. Our new complete gravity torque map of NGC 4579 makes it possible to quantify the efficiency of angular momentum transport from $r \sim$ a few kpc down to the inner $r \sim 50 \mathrm{pc}$ around the AGN and search for signatures of secular evolution in the accretion process. This is a key to testing the fueling scenario described by GB05. In particular, by studying the different spatial scales of the disk we can assess if dynamical decoupling is at work.

We will assume a distance to NGC 4579 of around $20 \mathrm{Mpc}$ for consistency with GB05, which implies $1^{\prime \prime} \simeq 100 \mathrm{pc}$. This is a good compromise between the different values reported in the literature ranging from $D=16.8 \mathrm{Mpc}$ (Tully \& Fisher 1988) to $D=22.6 \mathrm{Mpc}$ (LEDA, 2006). Similarly to GB05 we will initially assume that the inclination angle and the position angle of NGC 4579's disk are $i=36^{\circ}$ and $\mathrm{PA}=95^{\circ}$, respectively, in rough agreement with previous determinations based on kinematical model fitting (e.g., Rubin et al. 1999; Koopmann et al. 2001; Daigle et al. 2006) and photometric model fitting (e.g., Young \& Currie 1998; Paturel et al. 2000). These parameters will be further discussed in Sect. 6. The basic observational parameters of NGC 4579 are listed in Table 1. We describe in Sect. 2 the observations used, including high-resolution $\mathrm{CO}$ and $\mathrm{HI}$ maps as well as ground-based NIR images of NGC 4579. Sections 3 to 6 describe the distribution and kinematics of molecular gas. We describe the stellar structure and star formation of NGC 4579 in Sect. 7. Section 8 computes from NIR images the gravitational potentials and forces, and deduces from the combined $\mathrm{CO}$ and HI maps the effective torques applied to the gas. From these torques, it is possible to derive timescales for gas flows and discuss whether gravity torques alone are efficient enough to feed the current activity in NGC 4579. The general implications of these results for the current understanding of AGN feeding are summarized in Sect. 9.

\section{Observations and reduction}

\subsection{Interferometer $\mathrm{CO}$ maps}

Observations of NGC 4579 were carried out as part of the NUGA survey conducted with the PdBI during two years and finished by February 2003. We used the ABCD set of configurations and the six antennas of the array in dual frequency mode to reach the highest spatial resolution $\left(<1^{\prime \prime}\right.$ at the highest frequency) but also to maximize sensitivity to all spatial frequencies in the maps. We observed simultaneously the emission of the $J=1-0$ and $J=2-1$ lines of CO in single fields of sizes $42^{\prime \prime}$ and $21^{\prime \prime}$, respectively, centered at $\alpha_{\mathrm{J} 2000}=12^{\mathrm{h}} 37^{\mathrm{m}} 43.58^{\mathrm{s}}$ and $\delta_{\mathrm{J} 2000}=11^{\circ} 49^{\prime} 02.5^{\prime \prime}$. The first results derived from the analysis of the $\mathrm{CO}(2-1)$ maps only were discussed by GB05. During the observations the spectral correlator was split in two halves centered at the transition rest frequencies corrected for the recession velocity initially assumed $v_{\mathrm{o}}(\mathrm{HEL})=1516 \mathrm{~km} \mathrm{~s}^{-1}$. The correlator configuration covers a bandwidth of $580 \mathrm{MHz}$ for each line, using four $160 \mathrm{MHz}$-wide units with an overlap of $20 \mathrm{MHz}$; this is equivalent to $1510 \mathrm{~km} \mathrm{~s}^{-1}\left(755 \mathrm{~km} \mathrm{~s}^{-1}\right)$ at $115 \mathrm{GHz}$ $(230 \mathrm{GHz})$. Visibilities were obtained using on-source integration times of $20 \mathrm{~min}$ framed by short ( $\sim 2 \mathrm{~min}$ ) phase and amplitude calibrations on the nearby quasars $1156+295$ and 3C 273 . The absolute flux scale in our maps was derived to a $10 \%$ accuracy based on the observations of primary calibrators whose fluxes were determined from a combined set of measurements obtained at the $30 \mathrm{~m}$ telescope and the PdBI array. The bandpass calibration was carried out using 3C 273 and is accurate to better than $5 \%$. The point source sensitivities derived from emission-free channels of $10 \mathrm{~km} \mathrm{~s}^{-1}$ width are $2.55 \mathrm{mJy}^{-1}$ in $\mathrm{CO}(1-0)$ and $5.44 \mathrm{mJy}_{\text {beam }}^{-1}$ in $\mathrm{CO}(2-1)$. Images of the continuum emission of the galaxy at $115 \mathrm{GHz}$ and $230 \mathrm{GHz}$ have been obtained by averaging those channels free of line emission.

The image reconstruction was done using standard IRAM/GAG software (Guilloteau \& Lucas 2000). We used natural weighting and no taper to generate the $1-0$ line maps with a field of view of $76.8^{\prime \prime}$ and $0.15^{\prime \prime}$ sampling; the corresponding synthesized beam is $2.0^{\prime \prime} \times 1.3^{\prime \prime}, \mathrm{PA}=26^{\circ}$. We also used natural weighting to generate $2-1$ maps with a field of view of $51.2^{\prime \prime}$ and $0.10^{\prime \prime}$ sampling; this enables us to achieve a spatial resolution $<1^{\prime \prime}\left(1.0^{\prime \prime} \times 0.6^{\prime \prime}, \mathrm{PA}=25^{\circ}\right)$. The conversion factors between $\mathrm{Jy}_{\text {beam }}{ }^{-1}$ and $\mathrm{K}$ are $36 \mathrm{~K} \mathrm{Jy}^{-1}$ beam at $115 \mathrm{GHz}$, and $40 \mathrm{~K} \mathrm{Jy}^{-1}$ beam at $230 \mathrm{GHz}$.

\subsection{IRAM-30 m CO observations and short spacing correction}

With the aim of including the short spacing correction in the final image, we have mapped the emission of the 2-1 and 1-0 lines of CO in NGC 4579 with the IRAM 30 m telescope. We have covered the central $28^{\prime \prime}(2.8 \mathrm{kpc})$ region of the galaxy using a grid of $5 \times 5$ points with a $7 "$ spacing. The area covered is larger than the primary beam size of the PdBI at $230 \mathrm{GHz}$, and a significant fraction $(\sim 70 \%)$ of the corresponding field of view at $115 \mathrm{GHz}$. Observations were carried out in two observing runs between July 2002 and June 2004. At $115 \mathrm{GHz}$ and $230 \mathrm{GHz}$, the telescope half-power beam widths are $22^{\prime \prime}$ and $12^{\prime \prime}$, respectively. The $3 \mathrm{~mm}$ and $1 \mathrm{~mm}$ receivers of the $30 \mathrm{~m}$ telescope were tuned to the redshifted frequencies of the lines. The backends were two $1 \mathrm{MHz}$ filter banks and auto-correlator spectrometers. The velocity range covered was $1340 \mathrm{~km} \mathrm{~s}^{-1}$ for the $3 \mathrm{~mm}$ lines and 670 (for the inner part) to 1200 (for the outer part) $\mathrm{km} \mathrm{s}^{-1}$ for the $1 \mathrm{~mm}$ lines. Typical system temperatures during the observations were $\sim 250 \mathrm{~K}$ at $3 \mathrm{~mm}$ and $\sim 600 \mathrm{~K}$ at $1 \mathrm{~mm}$. All receivers were used in single side-band mode (SSB), with a high rejection of the image band: $>15 \mathrm{~dB}$ at $1 \mathrm{~mm}$ and $>20 \mathrm{~dB}$ at $3 \mathrm{~mm}$. The latter assures that the calibration accuracy for the bulk of our data is better than $20 \%$. Throughout the paper, velocity-integrated line intensities $(I)$ are given in antenna temperature scale, $T_{\mathrm{a}}^{*}$. The $T_{\mathrm{a}}^{*}$ scale relates to the main beam temperature scale, $T_{\mathrm{mb}}$, by the equation $T_{\mathrm{mb}}=\left(F_{\text {eff }} / B_{\text {eff }}\right) T_{\mathrm{a}}^{*}$, where $F_{\text {eff }}$ and $B_{\text {eff }}$ are the forward and beam efficiencies of the telescope at a given frequency. For the IRAM $30 \mathrm{~m}$ telescope $F_{\text {eff }} / B_{\text {eff }}=1.27(1.75)$ at $115 \mathrm{GHz}$ $(230 \mathrm{GHz})$ and $S / T_{\mathrm{mb}}=4.95 \mathrm{Jy} \mathrm{K}^{-1}$. Wobbler switching mode 

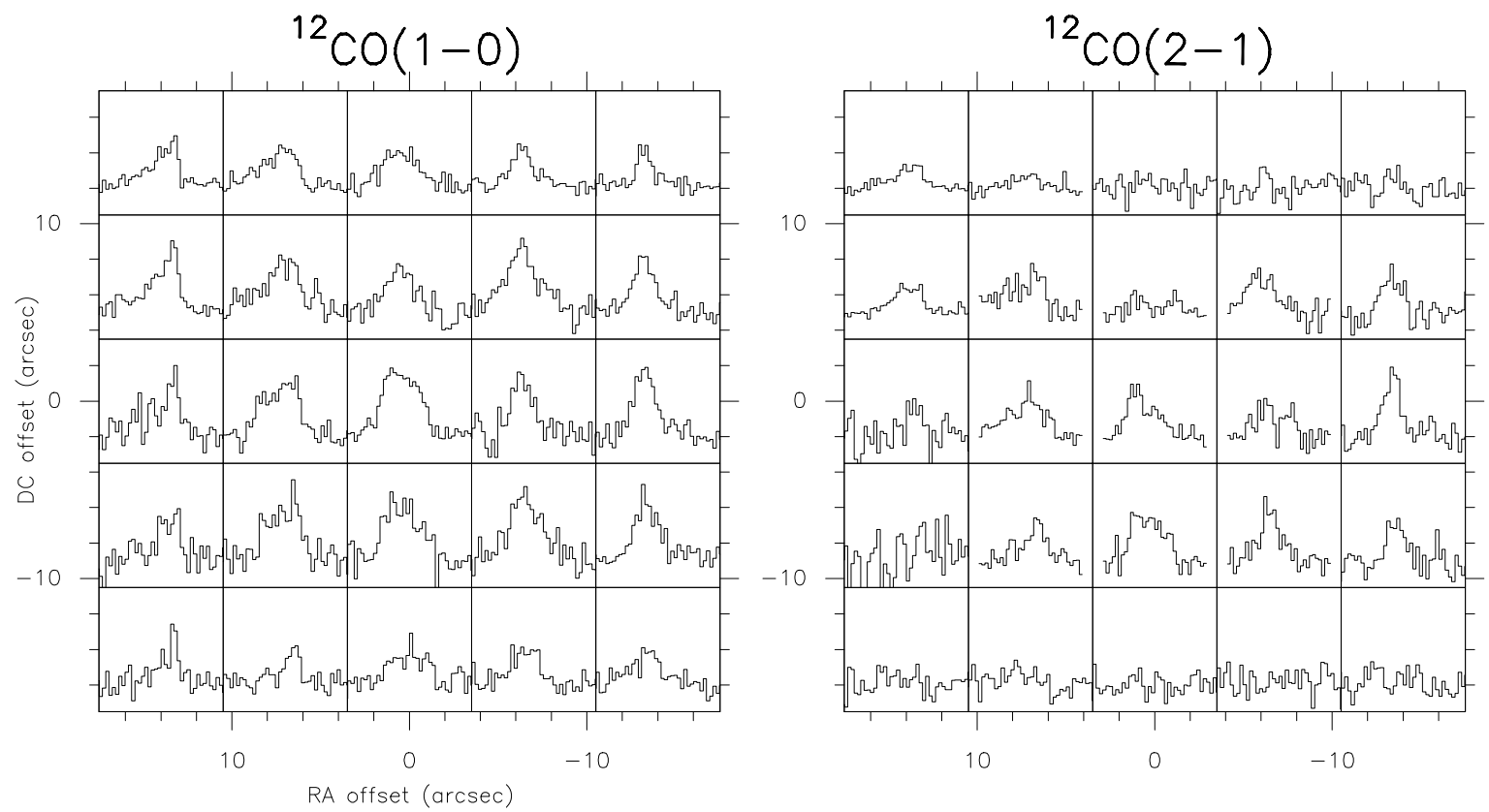

Fig. 1. a) (Left panel) $\mathrm{CO}(1-0)$ line emission profiles observed with the IRAM $30 \mathrm{~m}$ telescope in NGC 4579 . We have observed with a $5 \times 5$-point grid and a 7" spacing. Offsets are relative to the AGN. Velocity scale goes from $-400 \mathrm{~km} \mathrm{~s}^{-1}$ to $400 \mathrm{~km} \mathrm{~s}^{-1}$ with respect to $v=v_{0}=1516 \mathrm{~km} \mathrm{~s}{ }^{-1}$ in HEL scale, and temperature scale (in $T_{a}^{*}$ ) goes from -30 to $100 \mathrm{mK}$. b) (Right panel) Same as a) but for the CO(2-1) line. Same velocity scale as in a); temperature scale from -40 to $150 \mathrm{mK}$. All spectra have been smoothed to a resolution of $10 \mathrm{~km} \mathrm{~s}^{-1}$.

was used to assure flat baselines, taking a reference position offset by $4^{\prime}$ in azimuth. The pointing was regularly checked on continuum sources and the accuracy was $3^{\prime \prime}$ rms.

Short spacings were included in the combined PdBI+30 m image using the SHORT-SPACE task available in the GILDAS software (Guilloteau \& Lucas 2000). The relative weights are chosen so as to guarantee that similar absolute weights are taken for the single-dish data and the interferometer data within a ring in the UV plane going from $1.25 D / \lambda$ to $2.5 D / \lambda(D=15 \mathrm{~m})$. In the combined map we recovered all the missing flux and simultaneously kept the spatial resolutions very close to those of the original PdBI maps. Throughout this paper we use the combined PdBI+30 m data cube, i.e., corrected by short spacings. We estimate that the old map including only the PdBI spacings published by GB05 recovers $\sim 65 \%$ of the total $\mathrm{CO}(1-0)$ flux measured with the new combined $\mathrm{PdBI}+30 \mathrm{~m}$ map. A similar retrieval factor is derived for the $\mathrm{CO}(2-1)$ line. The $1-\sigma$ noise levels in $10 \mathrm{~km} \mathrm{~s}^{-1}$-wide channels for the PdBI+30 m images are $2.3 \mathrm{mJy}$ beam $^{-1}$ in $\mathrm{CO}(1-0)$ and $4.6 \mathrm{mJy} \mathrm{beam}^{-1}$ in $\mathrm{CO}(2-1)$.

The $30 \mathrm{~m}$ maps probe the $\mathrm{CO}$ emission of molecular gas in the central $r \sim 1.4 \mathrm{kpc}$ of NGC 4579 (Fig. 1). The emission is spatially resolved in $\mathrm{CO}(2-1)$ and is suggestive of an elongated gas distribution, mostly along the east-west axis. This picture is confirmed by the higher resolution interferometer data (see Sect. 4).

\subsection{H। maps}

The $21 \mathrm{~cm}$ HI line emission in NGC 4579 was observed in 2003 and 2004 using the NRAO Very Large Array (VLA) in its C and D-configurations. A first analysis of the results obtained in NGC 4579 have been published by Haan et al. (2008a) as part of a comprehensive spectroscopic imaging HI survey conducted in a sample of 16 LLAGNs including all NUGA galaxies. This HI survey reaches a moderate spatial resolution $\left(\sim 30-40^{\prime \prime}\right)$ and high sensitivity (3- $\sigma$ detection limit of $\leq 10^{19} \mathrm{~cm}^{-2}$ ). Calibration procedures followed are fully described in Haan et al. (2008a). The data from this HI survey have been recently the basis of an overall study of the in/out-flow rates in 7 NUGA galaxies (Haan et al. 2008b). The HI maps of NGC 4579 used in this paper have been produced by robustly weighting the imaging data in order to maximize spatial resolution while maintaining high sensitivity. With this procedure we achieve a spatial resolution of $23.1^{\prime \prime} \times 21.6^{\prime \prime}$ and a $1-\sigma$ sensitivity limit of $0.51 \mathrm{mJy}$ beam $^{-1}$. Analysis was done with the Groningen Image Processing SYstem (GIPSY) as described by Haan et al. (2008a). The channel maps were combined to derive an HI intensity map with a flux threshold of $3 \sigma$. The HI emission in the disk of NGC 4579 lies well inside the primary beam field of view of $30^{\prime}$; therefore no attempt has been made to correct for primary beam attenuation.

\subsection{Optical, UV and IR images}

We used several optical, UV and IR images of NGC 4579 to study the star formation pattern and the stellar structure of the disk of the galaxy as described below.

We first acquired from the HST archive $^{1}$ four broadband WFPC2 images of NGC 4579, including F502N ([OIII]), F658N $(\mathrm{H} \alpha)$, F547M, and F791W. Continuum-subtracted $\mathrm{H} \alpha$ and $[\mathrm{OIII}]$ emission line images were created by subtracting the associated continuum-band image, as described in Pogge et al. (2000). The optical images were combined using (crreject) to eliminate cosmic rays, and calibrated according to Holtzman et al. (1995). Sky values were assumed to be zero since the galaxy filled the WFPC2 frames, an assumption which makes an error of $\sim 0.1$ mag at most, in the corner of the images.

1 Based on observations made with the NASA/ESA Hubble Space
Telescope, obtained from the data archive at the Space Telescope
Science Institute. STScI is operated by the Association of Universities for Research in Astronomy, Inc. under NASA contract NAS 5-26555. 


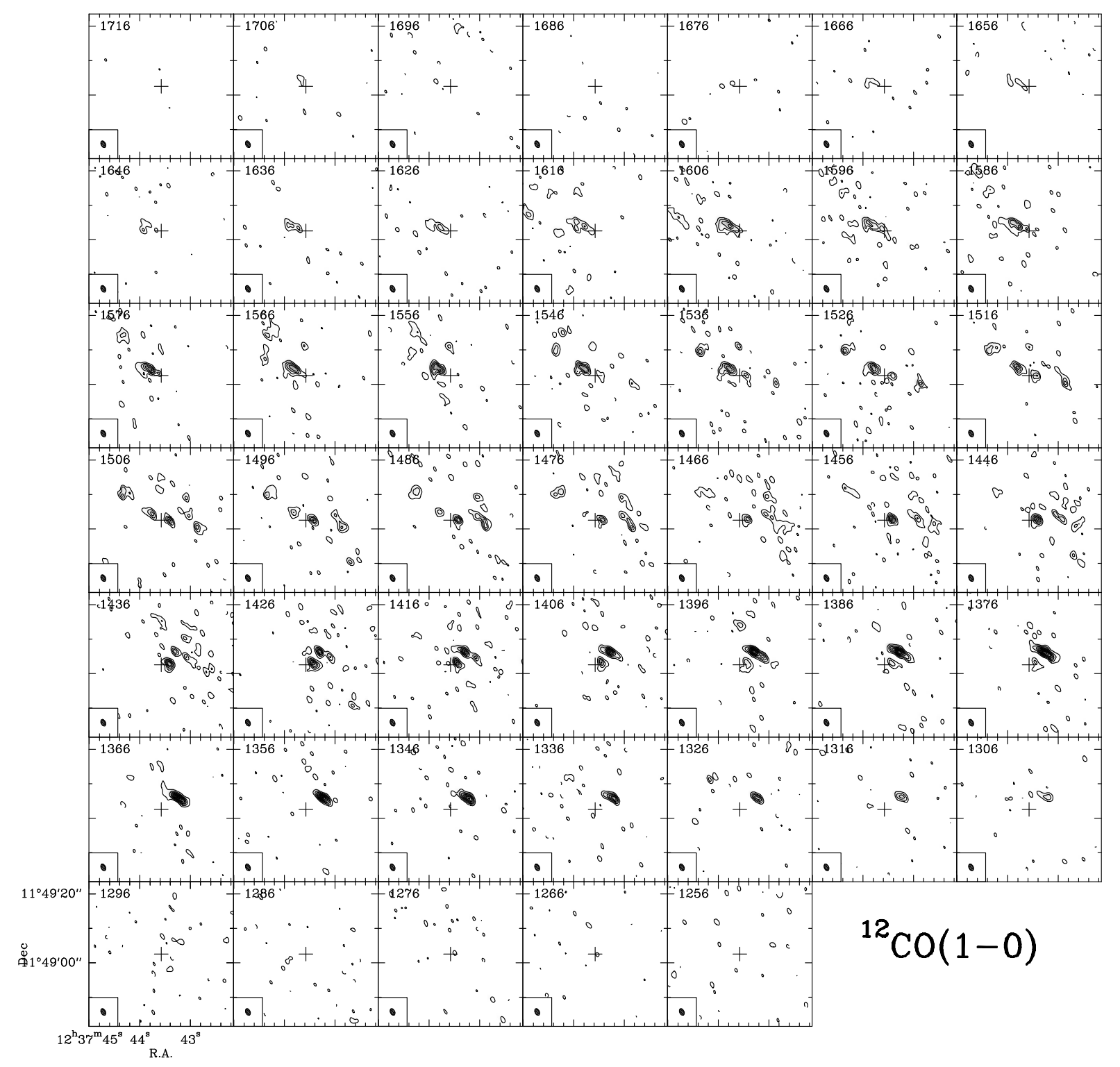

Fig. 2. ${ }^{12} \mathrm{CO}$ velocity-channel maps observed with the PdBI in the nucleus of NGC 4579 with a spatial resolution of $2.0^{\prime \prime} \times 1.3^{\prime \prime}$ at PA $=26^{\circ}($ beam is plotted as a filled ellipse in the bottom left corner of each panel). We show a field of view of $42^{\prime \prime}$, i.e. the diameter of the primary beam at $115 \mathrm{GHz}$. The phase tracking center is indicated by a cross at $\alpha_{2000}=12^{\mathrm{h}} 37^{\mathrm{m}} 43.58^{\mathrm{s}}$ and $\delta_{2000}=11^{\circ} 49^{\prime} 02.5^{\prime \prime}$. Velocity-channels are displayed from $v=1256 \mathrm{~km} \mathrm{~s}^{-1}$ to $v=1716 \mathrm{~km} \mathrm{~s}^{-1}$ in steps of $10 \mathrm{~km} \mathrm{~s}^{-1}$. Velocities are in HEL scale. Contour levels are $-3 \sigma, 3 \sigma$ to $24 \sigma$ in steps of $3 \sigma$ where the 1 -sigma $\mathrm{rms} \sigma=2.3 \mathrm{mJy}_{\text {beam }}{ }^{-1}$. There are very few pixels with negative fluxes below $-3 \sigma$ inside the field-of-view.

We used the ultraviolet (UV) image of NGC 4579 obtained with the Hubble Space Telescope's Advance Camera for Surveys (ACS) and its high-resolution channel detector (HRC) that provide $\sim 0.028^{\prime \prime} \times 0.025^{\prime \prime} /$ pixel spatial resolution. This image corresponds to the $F 330 \mathrm{~W}$ band whose central wavelength is $\sim 3300 \AA$ (Maoz et al. 2005). The image is available already reduced with the Space Telescope Science Institute (STScI) pipeline.

In addition, we also include the far-ultraviolet (FUV) image of the GALEX satellite, whose band is centered at $\lambda_{\text {eff }}=$ $1516 \AA$. This image has been obtained with a total exposure time of $1586 \mathrm{~s}$ and covers a square region on the sky of size $\sim 5800^{\prime \prime} \times$ $5800^{\prime \prime}$. The image is available already reduced with a slightly modified version of the GALEX data pipeline (Gil de Paz et al. 2007), expressed in intensity units and sky-subtracted.

IRAC images of NGC 4579 in bands $3.6 \mu \mathrm{m}$ and $8 \mu \mathrm{m}$ were retrieved from the Spitzer archive (Kennicutt et al. 2003). These images cover a region of the sky of size $\sim 1400^{\prime \prime} \times 1500^{\prime \prime}$ and have pixel size of $0.75^{\prime \prime}$. Images are reduced with the SINGS IRAC pipeline and are calibrated in $\mathrm{MJy} \mathrm{s}^{-1}$ units. We have recentered on the AGN and deprojected onto the galaxy plane both images to superpose these IR images onto the HI map.

Unlike GB05, where we used the HST I-band image to derive the stellar potential, here we adopt the $K$-band image obtained with the INGRID NIR camera of the William Herschel Telescope (WHT) by Knapen et al. (2003). The field of view provided by the camera is $4.2^{\prime} \times 4.2^{\prime}$ with a pixel size of $0.242^{\prime \prime}$. The details of the data reduction and calibration procedures for this image are described in Knapen et al. (2003).

\section{Dynamical center and position of the AGN}

Figures 2 and 3 show the velocity-channel maps of $\mathrm{CO}(1-0)$ and $\mathrm{CO}(2-1)$ emission in the central region of NGC 4579 , 


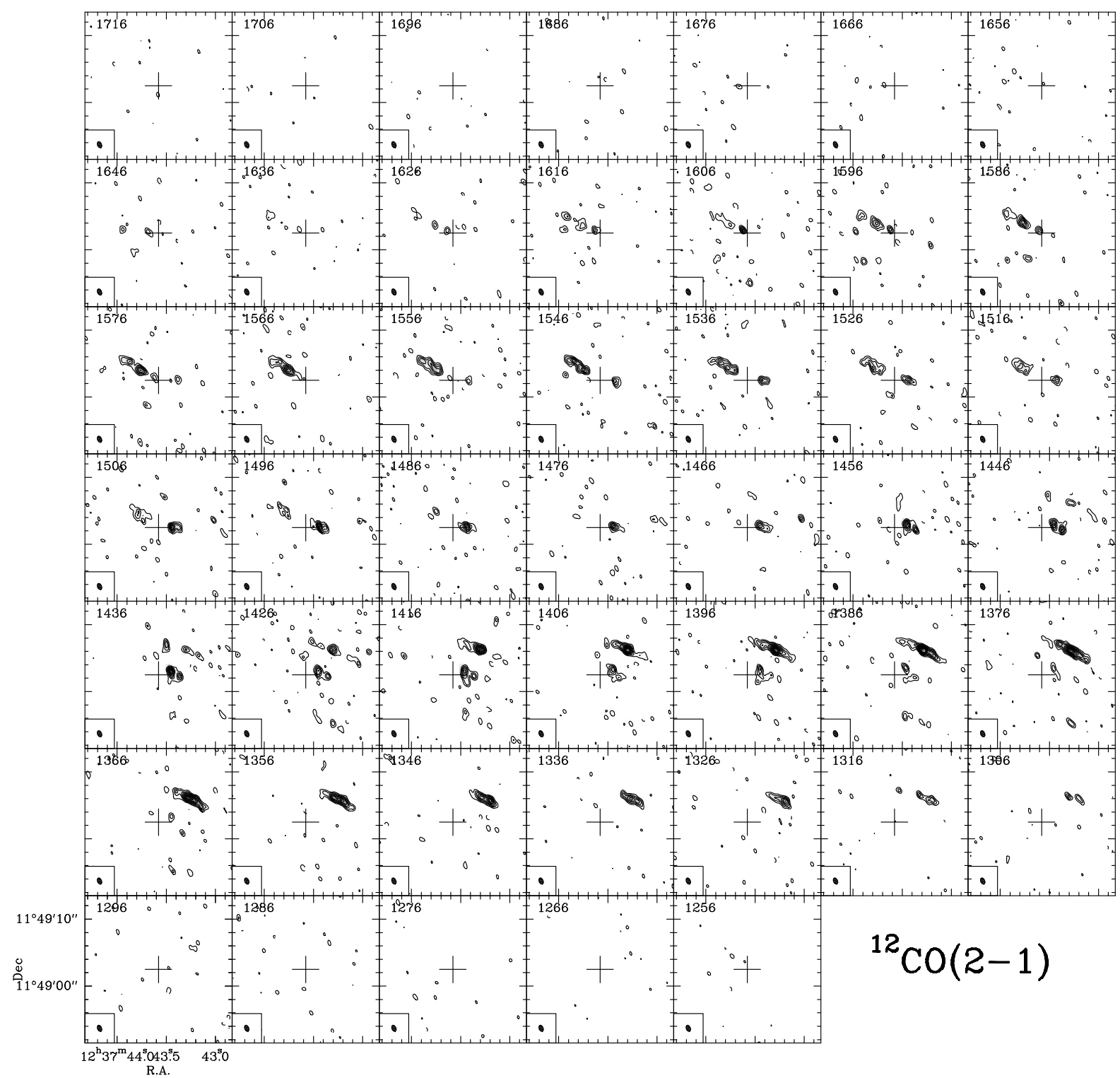

Fig. 3. Same as Fig. 2 but for the $2-1$ line of CO. Spatial resolution reaches $1.0^{\prime \prime} \times 0.6^{\prime \prime}$ at PA $=25^{\circ}$ (beam is plotted as a filled ellipse in the bottom left corner of each panel). We show a field of view of $21^{\prime \prime}$, i.e. diameter of the primary beam at $230 \mathrm{GHz}$. Velocity-channels are displayed from $v=1256 \mathrm{~km} \mathrm{~s}^{-1}$ to $v=1716 \mathrm{~km} \mathrm{~s}^{-1}$ in steps of $10 \mathrm{~km} \mathrm{~s}^{-1}$, with same reference as used in Fig. 2. Contour levels are $-3 \sigma, 3 \sigma$ to $19 \sigma$ in steps of $2 \sigma$ where the 1 -sigma rms $\sigma=4.6 \mathrm{mJy}_{\text {beam }}{ }^{-1}$. There are very few pixels with negative fluxes below $-3 \sigma$ inside the field-of-view.

respectively. The observed kinematical pattern is characteristic of a spatially resolved rotating structure of $\sim 2 \mathrm{kpc}$-radius. We detect extended emission above $3 \sigma$ levels from $v$ (HEL) $=$ 1296 to $1666 \mathrm{~km} \mathrm{~s}^{-1}$ in the $1-0$ line; this indicates that the overall $\mathrm{CO}(1-0)$ emission is centered around $v(\mathrm{HEL}) \sim 1480 \pm$ $10 \mathrm{~km} \mathrm{~s}^{-1}$. The corresponding velocity range for the $2-1$ line is $v(\mathrm{HEL})=1306$ to $1646 \mathrm{~km} \mathrm{~s}^{-1}$, i.e., the global emission is centered around $v(\mathrm{HEL}) \sim 1476 \pm 10 \mathrm{~km} \mathrm{~s}^{-1}$. The CO $(1-0)$ emission integrated over the central $r \sim 2.5 \mathrm{kpc}$ region mapped with the $30 \mathrm{~m}$ telescope also shows a similar velocity centroid $v(\mathrm{HEL}) \sim 1487 \pm 20 \mathrm{~km} \mathrm{~s}^{-1}$ (Fig. 4).

We derive the position of the $\mathrm{AGN}$, at $(\Delta \alpha, \Delta \delta)=$ $\left(-0.9^{\prime \prime}, 3^{\prime \prime}\right)=\left(\alpha_{\mathrm{J} 2000}=12^{\mathrm{h}} 37^{\mathrm{m}} 43.52^{\mathrm{s}}, \delta_{\mathrm{J} 2000}=11^{\circ} 49^{\prime} 05.54^{\prime \prime}\right)$, from the detection of a point source in continuum emission at $115 \mathrm{GHz}$ (with a flux of $11.5 \pm 0.3 \mathrm{mJy}$ ) and $230 \mathrm{GHz}$ (with a flux of $11.0 \pm 1 \mathrm{mJy}$, see Fig. 5). Within the errors, this value coincides with previous determinations based on multi-frequency very-long-baseline interferometry (VLBI) observations of the non-thermal continuum emission of NGC 4579 (Anderson et al. 2004; Krips et al. 2007). As discussed by Krips et al. (2007), the spectral index of the continuum emission from $1 \mathrm{GHz}$ to $230 \mathrm{GHz}$ indicates that the spectrum of the AGN source is flat or slightly inverted, as frequently found in LLAGNs. In the following we adopt the AGN position as the dynamical center. This choice maximizes the symmetry of the global velocity field derived from the two CO lines (see Sect. 6.1).

Weak but still statistically significant emission has been detected in the 2-1 and 1-0 lines of CO coming from a spatially unresolved clump at the position of the AGN (see Fig. 5). Emission is detected at $\mathrm{a} \geq 4-\sigma$ level in integrated intensities over a velocity range $\Delta v \sim 150 \mathrm{~km} \mathrm{~s}^{-1}$. This suggests the existence of a compact molecular source of radius $r \leq 40 \mathrm{pc}$, an upper limit determined by the beam size at $230 \mathrm{GHz}$. The velocity centroid of the $\mathrm{CO}$ emission detected in the central clump is $v_{\mathrm{AGN}} \sim$ $1466 \pm 20 \mathrm{~km} \mathrm{~s}^{-1}$ in the $2-1$ line. The $1-0$ spectrum towards the $\mathrm{AGN}$ is $20 \mathrm{~km} \mathrm{~s}^{-1}$ blueshifted with respect to this value. In 


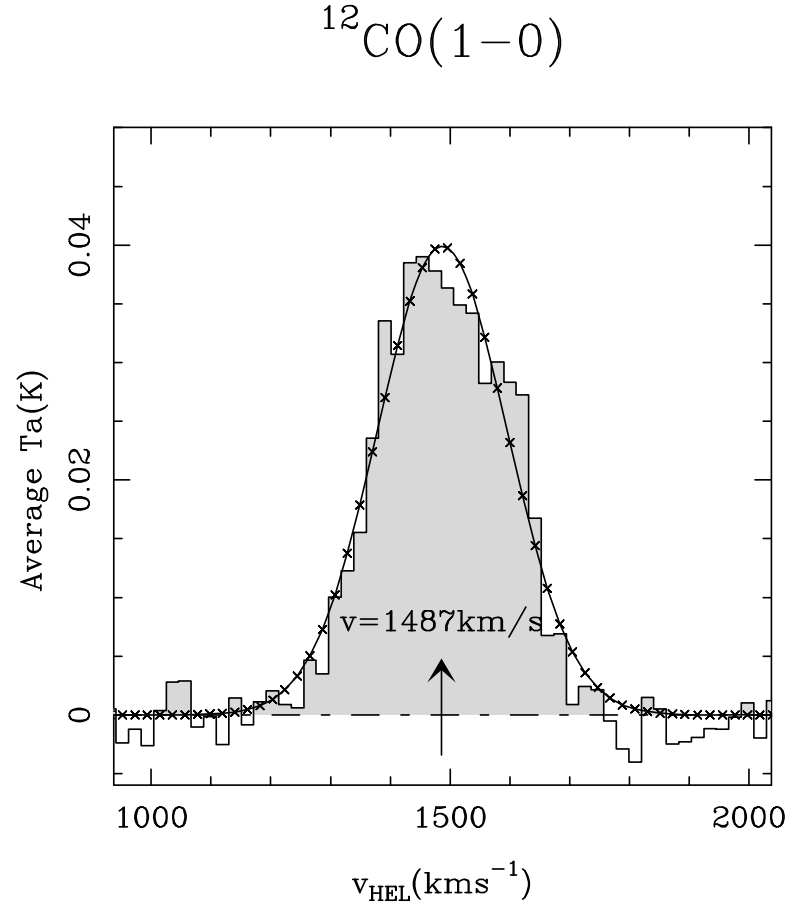

Fig. 4. $\mathrm{CO}(1-0)$ line emission averaged over the area mapped with the $30 \mathrm{~m}$ telescope towards NGC 4579 .

the following we adopt $v_{\mathrm{AGN}}$ derived from the $\mathrm{CO}(2-1)$ spectrum as the best estimate of $v_{\text {sys }}$. We expect the kinematics of the central clump to be dominated by the axisymmetric gravitational potential of the galaxy on these scales. In addition, this value coincides within the errors with the centroid of velocities of the global $\mathrm{CO}$ emission on kpc scales, independently derived from the PdBI and $30 \mathrm{~m}$ maps used in this work. It also coincides with previous estimates of $v_{\text {sys }}$ based on single-dish CO data of NGC 4579 (Elfhag et al. 1996; Kenney \& Young 1988).

The adopted systemic velocity derived from $\mathrm{CO}$ is $\sim 50 \mathrm{~km} \mathrm{~s}^{-1}$ blueshifted with respect to the value determined from HI $\left(v_{\text {sys }}^{\mathrm{HEL}}=1519 \pm 10 \mathrm{~km} \mathrm{~s}^{-1}\right.$ from RC3 $)$. The reasons for this discrepancy might be found in the interaction history of the galaxy. NGC 4579 is one of the most HI deficient galaxies of the Virgo cluster and its HI disk is known to have suffered heavy ram-pressure stripping in the past well within the optical disk (Kenney \& Young 1986, 1989; Haan et al. 2008a). Therefore the global kinematics of the HI disk are expected to have been perturbed due to the ICM-ISM interaction. However, molecular gas is known to be much less affected by ram-pressure (e.g., Kenney \& Young 1986, 1989; Vollmer et al. 2001). In such a scenario, the disagreement between the $\mathrm{HI}$ and $\mathrm{CO}$ values for $v_{\text {sys }}$ is not surprising if the HI kinematics retain some memory of the interaction. Since $\mathrm{HI}$ is virtually depleted inside $r \sim 2 \mathrm{kpc}$, the value of $v_{\text {sys }}$ derived from $\mathrm{HI}$ is sensitive to emission that comes from the outer disk of the galaxy, where the effects of ram-pressure stripping are known to be more dramatic. We therefore conclude that the value of $v_{\text {sys }}$ based on $\mathrm{CO}$, besides symmetrizing the velocity field of the gas inside $r \sim 2 \mathrm{kpc}$, is more representative than the value derived from HI. Other estimates of $v_{\text {sys }}$, based on the study of optical emission lines or stellar kinematics, give values intermediate between those of $\mathrm{CO}$ and $\mathrm{HI}$ (e.g., Huchra et al. 1982; Héraudeau \& Simien 1998).

The eastern (western) side of the $\mathrm{CO}$ distribution is globally red (blue)-shifted with respect to $v_{\text {sys }}$ (Figs. 2 and 3 ). This agrees qualitatively with the kinematical major axis of the circumnuclear disk being oriented roughly along the RA-axis, an orientation similar to that of the outer disk $(r>5-10 \mathrm{kpc})$ probed by $\mathrm{HI}$ and $\mathrm{H} \alpha$ emission (Guhathakurta et al. 1988; Chemin et al. 2006; Haan et al. 2008a). The global kinematics are perturbed by non-circular motions (see Sect. 6). In this paper we will adopt a common $\mathrm{PA}=95^{\circ}$ at all radii in rough agreement with the values derived from $\mathrm{H} \alpha$ and $\mathrm{HI}$ kinematics, as well as with isophotal fitting of galaxy images. This choice of PA will be motivated further in Sect. 6.1.

\section{Molecular gas distribution}

The velocity-integrated $\mathrm{CO}(1-0)$ flux obtained in the combined PdBI+30 m map within the 42" primary beam field of the $\mathrm{PdBI}$ is $S_{\mathrm{CO}}=150 \mathrm{Jy} \mathrm{km} \mathrm{s}^{-1}$. This is $\sim 90 \%$ of the total flux detected by Kenney \& Young (1988) towards the central position of a raster map of NGC 4579, made along the major axis of the galaxy with the Five College Radio Astronomy Observatory (FCRAO) telescope ${ }^{2}$. Assuming a CO-to- $\mathrm{H}_{2}$ conversion factor typical of galaxy nuclei $X=N\left(\mathrm{H}_{2}\right) / I_{\mathrm{CO}}=2.3 \times$ $10^{20} \mathrm{~cm}^{-2} \mathrm{~K}^{-1} \mathrm{~km}^{-1} \mathrm{~s}$ (Solomon \& Barrett 1991), the total $\mathrm{H}_{2}$ mass derived from the $\mathrm{PdBI}+30 \mathrm{~m}$ map within the $42^{\prime \prime} \mathrm{PdBI}$ field of view is $M\left(\mathrm{H}_{2}\right) \sim 5 \times 10^{8} M_{\odot}$. Including the mass of helium, the corresponding total molecular gas mass is $M_{\text {gas }}=M\left(\mathrm{H}_{2}+\mathrm{He}\right)=1.36 \times M\left(\mathrm{H}_{2}\right) \sim 7 \times 10^{8} M_{\odot}$. The distribution of molecular gas is best seen in Figs. 6a and b, which show the CO intensity maps obtained by integrating the emission in velocity channels from $v=1256$ to $1716 \mathrm{~km} \mathrm{~s}^{-1}$ using a 3- $\sigma$ clipping.

The sensitivity of the $\mathrm{CO}(1-0)$ map is reduced beyond the primary beam radius at this frequency $r=21^{\prime \prime}(\sim 2 \mathrm{kpc})$. This explains why we do not see in our maps the large-scale twoarm spiral structure detected in the single-dish $\mathrm{CO}(1-0)$ map of Kuno et al. (2007). This large-scale spiral develops from $r \sim$ $4 \mathrm{kpc}$ to $r \sim 10 \mathrm{kpc}$ and shows a good correspondence with the HI pseudo-ring detected by Haan et al. (2008a, see Fig. 12a). Normalized to a distance $D=20 \mathrm{Mpc}$, we estimate that the total molecular gas mass inside $r \sim 10 \mathrm{kpc}$ amounts to $M_{\mathrm{gas}} \sim$ $4 \times 10^{9} M_{\odot}$.

The integrated-intensity contours are overlaid on the HST $V-I$ image in Fig. 7 and on the [OIII] emission in Fig. 8. It can be seen that there is little molecular gas at $r<200 \mathrm{pc}$ from the central engine. The most massive gas complexes, of a few $\sim 10^{6} M_{\odot}$, lie east and north of the nucleus at $r \sim 150 \mathrm{pc}$ (complexes $\mathrm{E}$ and $\mathrm{N}$ in Fig. 8, respectively). Complexes $\mathrm{E}$ and $\mathrm{N}$ are connected by an arc of low-level $\mathrm{CO}(2-1)$ emission (complexes $\mathrm{E}^{\prime}$ and $\mathrm{N}^{\prime}$ in Fig. 8).

\subsection{Gas components}

Inside $r \sim 2 \mathrm{kpc}$ we identify in the PdBI maps three main components in the distribution of the 2-1 and 1-0 line emission:

- The central disk: the 2-1 line map of Fig. 6b gives a sharp view of the distribution of molecular gas at radii $r<200 \mathrm{pc}$. The molecular clumps (complexes $\mathrm{N}, \mathrm{N}^{\prime}, \mathrm{E}^{\prime}, \mathrm{E}$ ) constitute together the molecular gas counterpart of a lopsided disklike structure that can be clearly identified as a dusty feature in the $V-I$ color HST image of the galaxy (Fig. 7), and as a filled-in disk in the $\mathrm{H} \alpha$ image (Fig. 13) discussed in Sect. 7.

\footnotetext{
2 Note that the two flux estimates are directly comparable as the FWHM of the FCRAO at $115 \mathrm{GHz}$ is $45^{\prime \prime}$, i.e., similar to the corresponding PdBI primary beam size at this frequency
} 

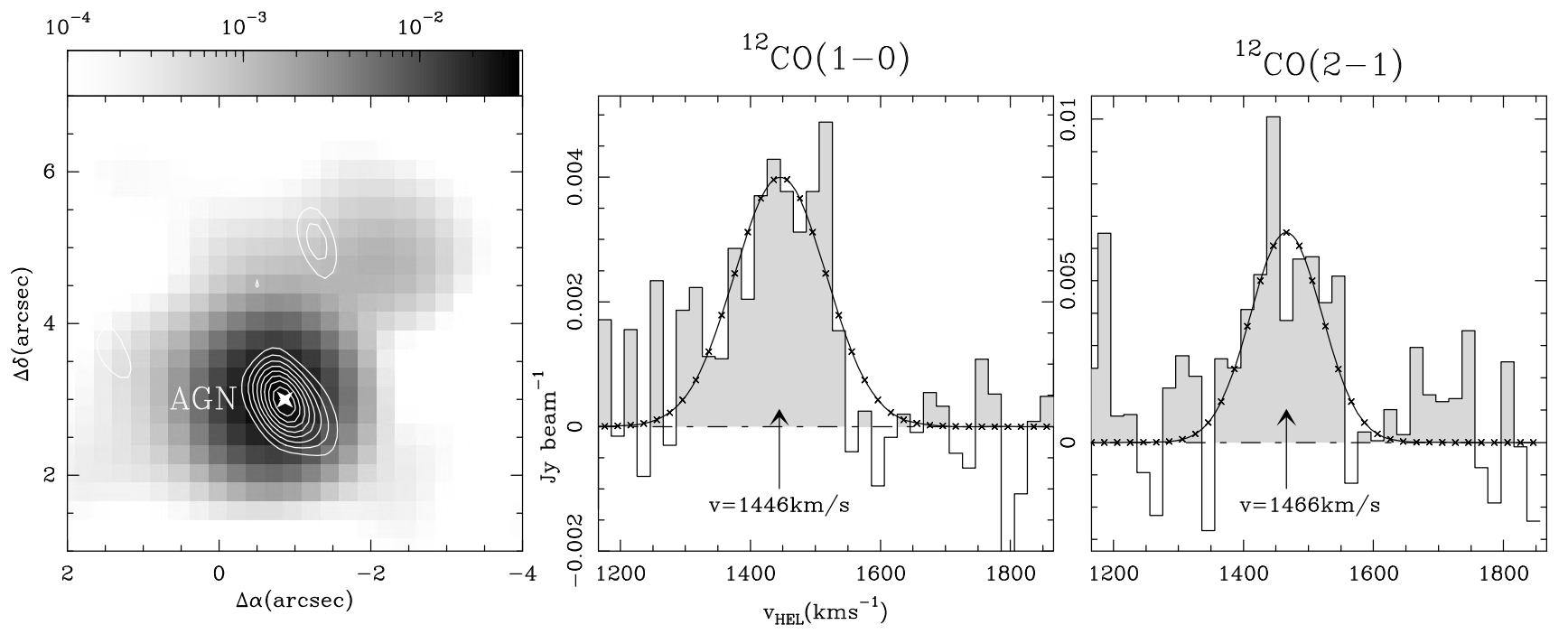

Fig. 5. a) (Left panel) The continuum map at $1 \mathrm{~mm}$ obtained with the PdBI (in contours from $20 \%$ to $100 \%$, in steps of $10 \%$ of the maximum $=$ $10 \mathrm{mJy}$ beam $^{-1}$ ) is overlaid on the VLA radio-continuum image at $6 \mathrm{~cm}$ (in grey scale) taken from Ho \& Ulvestad (2001). The AGN is clearly identified with a non-thermal point source. $(\Delta \alpha, \Delta \delta)$-offsets are here relative to the phase tracking center. b) (Middle panel) We show the emission associated with the AGN source observed in the $\mathrm{CO}(1-0)$ line at PdBI. c) (Right panel) The same as b) but for $\mathrm{CO}(2-1)$.

The AGN lies close to the southwestern edge of this central disk. As discussed in Sect. 3, there is also weak CO emission related to the AGN; this corresponds to a compact molecular gas structure (torus?) of $M_{\text {gas }}=M\left(\mathrm{H}_{2}+\mathrm{He}\right) \sim 10^{6} M_{\odot}$.

- The inner spiral arms: the bulk $(>80 \%)$ of the molecular gas as traced by the two CO lines is piled up in two highly contrasted spiral lanes that lie at the leading edges of the $\sim 12 \mathrm{kpc}$-diameter bar, identified in the $K$-band image of Knapen et al. (2003; see also Eskridge et al. 2002; and Jarrett et al. 2003). This geometry is discussed more at length in Sect. 7, and is also illustrated in Figs. 12b and c, which show the deprojected version of the CO maps overlaid on the $K$ band image of the galaxy. The CO arms develop from $r \sim$ $300 \mathrm{pc}$ to $r \sim 1.3 \mathrm{kpc}$. At closer examination, it can be seen that the CO spiral lane stretching north of the AGN (hereafter denoted as $\mathrm{N}$-arm) is more regular and better delineated than its southern counterpart (hereafter referred to as $\mathrm{S}$-arm). Moreover, the inner segment of the $\mathrm{N}$-arm is significantly closer to the AGN compared to that of the S-arm (Fig. 7). As discussed in Sect. 7 (see also Fig. 13), a similar asymmetry is observed in $\mathrm{H} \alpha$ emission. Furthermore, the $\mathrm{N}$-arm is well correlated with a northern dust lane characterized by a red $V-I$ color (Fig. 7). In contrast, the S-arm shows little, if any, color signature in the $V-I$ color image of Fig. 7. This overall north-south asymmetry in the color image can be partly explained if we assume that the northern side of the disk is the near side of the galaxy. Nevertheless, the differences in the morphology of the $\mathrm{N}$-arm and the $\mathrm{S}$-arm, revealed by $\mathrm{CO}$ and $\mathrm{H} \alpha$, are to a large extent intrinsic and thus cannot be explained only by a projection effect.

- The outer arc: further out, to the north, there is a chain of emission clumps detected in $\mathrm{CO}(1-0)$ (hereafter referred to as outer arc or $\mathrm{O}$-arc). This component, only detected in the 1-0 line, has no clear southern equivalent. As shown in Fig. 7, the O-arc is well correlated with a chain of gas spurs characterized by a red $V-I$ color. Furthermore, the $\mathrm{O}$-arc is also detected in $\mathrm{H} \alpha$ emission (see Fig. 13 and discussion in Sect. 7); this morphology, first described by Pogge (1989) as unusual, resembles a one-sided, partially filled-in, ellipsoidal ring.
In summary, the bulk of the gas response in the disk of NGC 4579, as traced by the PdBI CO maps described above, follows the expected gas distribution induced by the bar potential of the galaxy. In particular, the inner spiral arms, morphologically an $m=2$ perturbation, appear as two offset gas lanes which are leading with respect to the bar. This configuration suggests that there is a spatially extended inner Lindblad resonance (ILR) region in the nucleus, i.e., leaving room for two ILRs. In terms of bar orbit structure, this result can be interpreted as an indication that there are stable stellar orbits whose major axis is perpendicular to the bar major axis ( $x_{2}$ orbits, e.g., Athanassoula 1992; Buta \& Combes 1996; Regan \& Teuben 2003).

\section{CO line ratios}

The value of the $2-1 / 1-0$ integrated intensity ratio $\left(R_{21}\right)$ is obtained after convolving the 2-1 map to the lower resolution of the 1-0 map, both maps including short spacings. $R_{21}$ ranges from $\sim 0.4$ to $\sim 1$ inside the observed region (in $T_{\mathrm{mb}} \mathrm{km} \mathrm{s}^{-1}$ units); the average ratio is $\sim 0.6$. This ratio is close to the canonical value observed in spiral disks and is typical of optically thick molecular gas clouds (e.g., Braine \& Combes 1992; García-Burillo et al. 1993). There is a radial trend in the excitation of the gas, as measured by the line ratio: close to the AGN, at $r<200 \mathrm{pc}$, $R_{21} \sim 0.8-1$ (over the central disk), whereas $R_{21} \sim 0.4-0.6$ at $r>$ $500 \mathrm{pc}$ (over the $\mathrm{S}$-arm, the $\mathrm{N}$-arm and the $\mathrm{O}$-arc). Hints of a similar trend are found in other NUGA targets (e.g., García-Burillo et al. 2003b) and can be interpreted as evidence of external heating of molecular clouds by X-rays in the vicinity of the AGN (Baker et al. 2003).

\section{Molecular gas kinematics}

\subsection{Rotation curve and dynamical mass}

We show in Fig. 9 the isovelocity contour maps obtained from the $\mathrm{CO}(1-0)$ and $\mathrm{CO}(2-1)$ data in the nucleus of NGC 4579 (applying a 4- $\sigma$ clipping). The kinematics of the molecular gas are consistent with those of a spatially resolved rotating structure. 


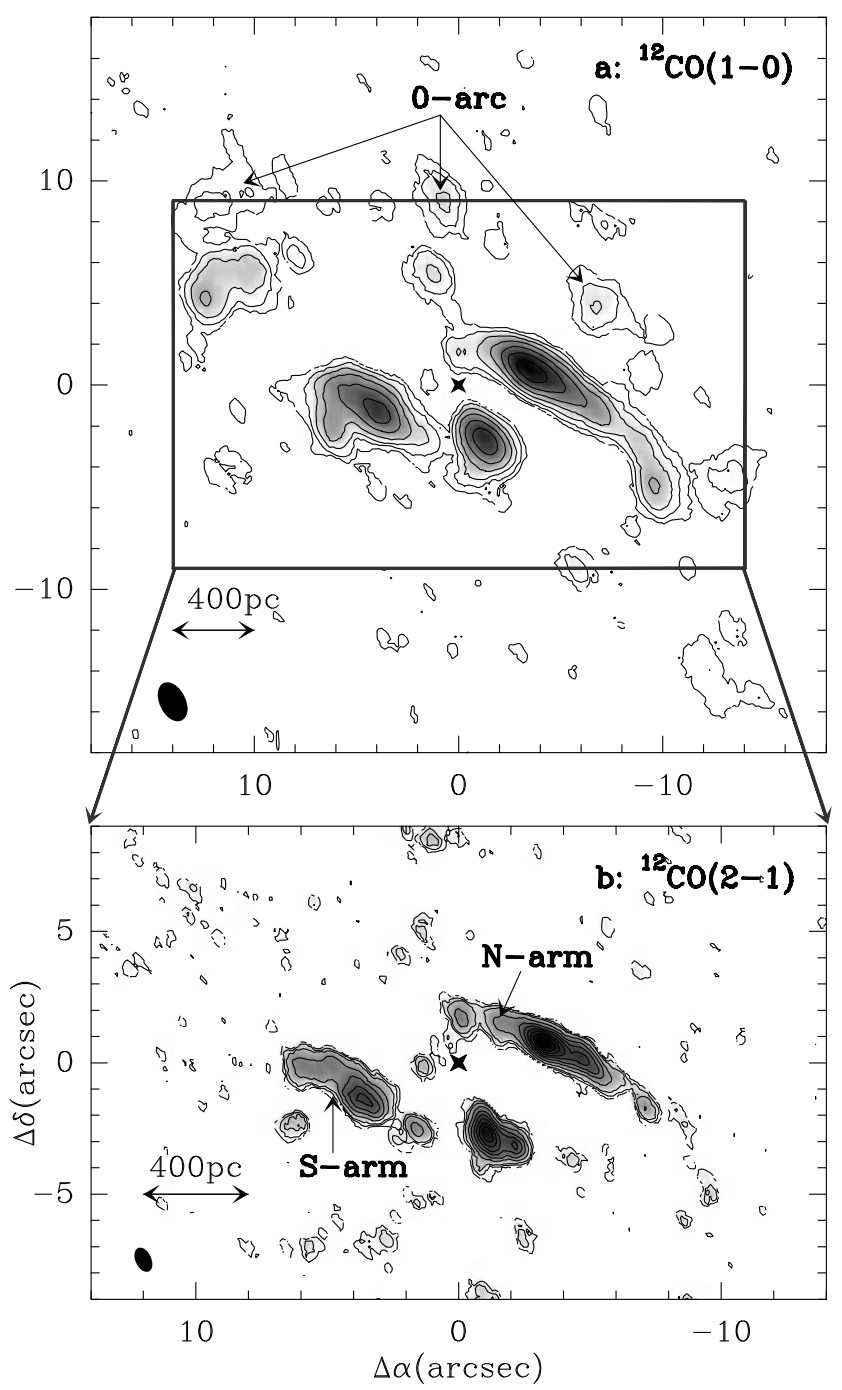

Fig. 6. a) The $\mathrm{CO}(1-0)$ integrated intensity map obtained with the PdBI in the nucleus of NGC 4579. The map is shown in grey scale with contour levels $0.2,0.5,1,1.7,2.6,3.7$ and $5 \mathrm{Jy} \mathrm{km} \mathrm{s}^{-1}$ beam $^{-1}$. The filled ellipse at the bottom left corner represents the $\mathrm{CO}$ beam size. $(\Delta \alpha, \Delta \delta)$ offsets are with respect to the location of the AGN (marked by the star): $\left(\mathrm{RA}_{2000}, \operatorname{Dec}_{2000}\right)=\left(12^{\mathrm{h}} 37^{\mathrm{m}} 43.52^{\mathrm{s}}, 11^{\circ} 49^{\prime} 05.5^{\prime \prime}\right)$. b) Same as a) but here for the $\mathrm{CO}(2-1)$. Contours levels from 0.3, 0.6, 1 to 9 in steps of $1 \mathrm{Jy} \mathrm{km} \mathrm{s}^{-1}$ beam ${ }^{-1}$. Positive intensity contours have been derived using a 3- $\sigma$ clipping on both data cubes. The position of the N-arm, S-arm and $\mathrm{O}$-arc, as defined in the text, are highlighted.

The sense of the rotation of the gas in the galaxy plane is counterclockwise assuming the northern side is the near side (see Sect. 4).

An estimate of the CO rotation curve $\left(v_{\text {rot }}\right)$ can be obtained from position-velocity $(p-v)$ diagrams taken along the major axis of NGC 4579 (Fig. 10), assuming that circular motions dominate the gas kinematics. We have fitted the major axis position angle by maximizing the line-of-sight velocity gradient within $r=2 \mathrm{kpc}$, and obtain $\mathrm{PA}=95 \pm 10^{\circ}$; this value is consistent within the errors with previous findings based on $\mathrm{H} \alpha$ and HI kinematics (Guhathakurta et al. 1988; Chemin et al. 2006; Haan et al. 2008a). It is also coincident with the PA given by isophotal fitting of optical images (Young \& Currie 1998; Paturel et al. 2000). To assess the validity of the adopted PA, we have made our own isophotal fitting of the $3.6 \mu \mathrm{m}$ IRAC image shown in Fig. 14; the value obtained from the fit confirms within the

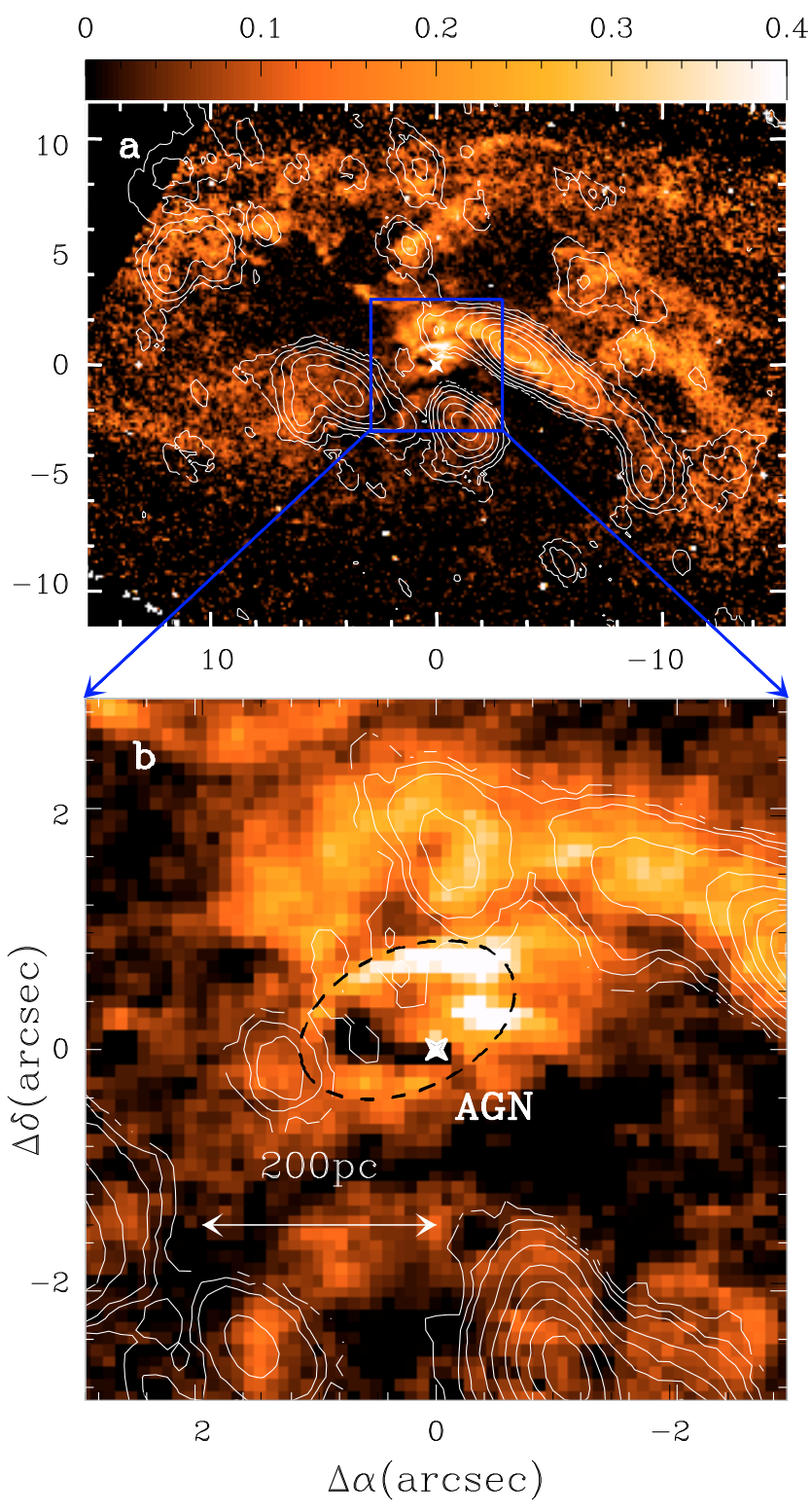

Fig. 7. a) The $\mathrm{CO}(1-0)$ integrated intensity map obtained with the PdBI (contour levels are $0.2,0.5,1.0,1.7,2.6,3.7$ and $5 \mathrm{Jy} \mathrm{km} \mathrm{s}^{-1}$ beam ${ }^{-1}$ ) is overlaid on the $V-I$ color image from HST (color scale) observed in the nucleus of NGC 4579. ( $\Delta \alpha, \Delta \delta)$-offsets are with respect to the location of the AGN (marked by the star). b) Same as a) for the $\mathrm{CO}(2-1)$ line with contour levels from $0.3,0.6,1.0$ to 9 in steps of $1.0 \mathrm{Jy} \mathrm{km} \mathrm{s}^{-1}$ beam $^{-1}$. A close-up view of the inner $200 \mathrm{pc}$ region shows a lopsided ring-like disk (highlighted by the dashed ellipse) with the AGN lying on its southwestern edge.

errors that $\mathrm{PA}=95 \pm 10^{\circ}$. Terminal velocities $\left(v_{\text {term }}\right)$ are derived by fitting multiple Gaussian profiles to the spectra across the $\mathrm{CO}$ major axis and selecting at each position the best estimate for $v_{\text {term }}$, taking into account velocity dispersion. During the fitting process we have masked the $\mathrm{CO}$ spectra between $0.5^{\prime \prime}(50 \mathrm{pc})$ and $2.5^{\prime \prime}(250 \mathrm{pc})$ east of the dynamical center because strong non-circular motions in this region make the gas apparently counter-rotate around the nucleus (see Fig. 10 and discussion in Sect. 6.2). For the same reasons, the data points from the $\mathrm{O}$-arc have not been included in the fit. We have confirmed the adopted inclination of $i=36 \pm 3^{\circ}$ through isophotal fitting of the $3.6 \mu \mathrm{m}$ IRAC image. The velocity centroids, corrected for inclination $i=36^{\circ}$, and referred to $v=v_{\text {sys }}$, give an 

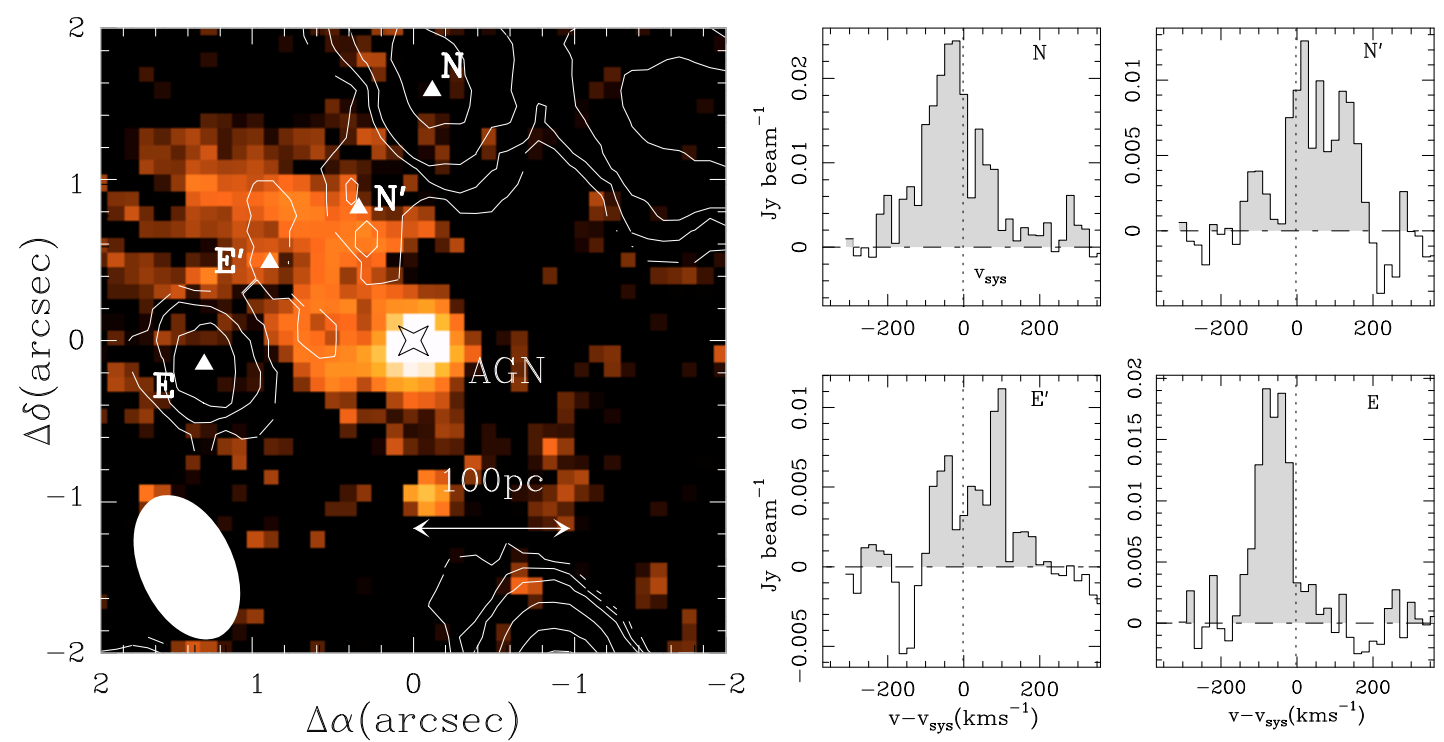

Fig. 8. a) (Left panel) Same contours for the $\mathrm{CO}(2-1)$ line as in Fig. 7 overlaid on the [OIII] line color image from HST of Pogge et al. (2000) (color scale) observed in the nucleus of NGC 4579. Complexes N, $\mathrm{N}^{\prime}, \mathrm{E}^{\prime}$ and $\mathrm{E}$ are highlighted. b) (Right panels) $\mathrm{CO}(2-1)$ line spectra observed towards complexes $\mathrm{N}, \mathrm{N}^{\prime}, \mathrm{E}^{\prime}$ and $\mathrm{E}$. Velocities are relative to $v_{\mathrm{sys}}$.

estimate of $v_{\text {rot }}$ for each offset along the major axis. To extend $v_{\text {rot }}$ to the outer radii of the galaxy we have included in the global fit the HI data points of Guhathakurta et al. (1988) and Haan et al. (2008a).

A simple rotation curve model can be fitted using the $\mathrm{CO}+\mathrm{HI}$ data from both sides of the major axis. We choose a rotation law characteristic of a logarithmic gravitational potential, described by

$v_{\text {rot }}(r)=\frac{v_{\max } r}{\sqrt{r^{2}+r_{\text {max }}^{2}}}$

where $v_{\max }$ is the maximum rotation velocity and $r_{\max }$ is a radial scale whose value controls the steepness of $v_{\text {rot }}$. The best fit gives $v_{\max }=295 \mathrm{~km} \mathrm{~s}^{-1}$ and $r_{\max }=530 \mathrm{pc}$ (Fig. 11). The $v_{\text {rot }}$ curve shows a gradual increase to $r \sim 0.65 \mathrm{kpc}$, where $v_{\text {rot }} \sim$ $240 \mathrm{~km} \mathrm{~s}^{-1}$. This roughly linear increase seen in the inner region is consistent with the trend in the stellar absorption line rotation curve (Palacios et al. 1997). From $r \sim 3 \mathrm{kpc}$ to the outer disk traced by HI, up to $r \sim 12 \mathrm{kpc}, v_{\text {rot }}$ remains roughly flat at $\sim 295 \mathrm{~km} \mathrm{~s}^{-1}$.

The mass inside a certain radius $r$ can be inferred using $M(r)=C \times r \times v_{\mathrm{rot}}^{2} / G$, where $G$ is the constant of gravity, $M(r)$ is the mass inside a sphere of radius $r$, and $C$ is a constant varying between 0.6 and 1, depending on the disk mass model assumed. If we take a value of $C=0.8$ intermediate between the values appropriate for spherical (1) and flat disk (0.6) distributions, $M(r=2 \mathrm{kpc}) \sim 4 \times 10^{10} M_{\odot}$. This implies that the molecular gas mass fraction inside $r=2 \mathrm{kpc}$ is $\sim 2 \%$. This fraction decreases to $\leq 0.6 \%$ at $r=50 \mathrm{pc}$.

From the best fit of $v_{\text {rot }}$, we have derived the frequency curves, shown in Fig. 11: $\Omega, \Omega \pm \kappa / 2$ and $\Omega-\kappa / 4$. These curves can be used to estimate the position of the main resonances in the disk in the context of the epicyclic approximation as discussed in Sect. 7, where we compare the morphology of the molecular disk to the distribution shown by other tracers of the ISM and the stellar structure in the disk of NGC 4579.

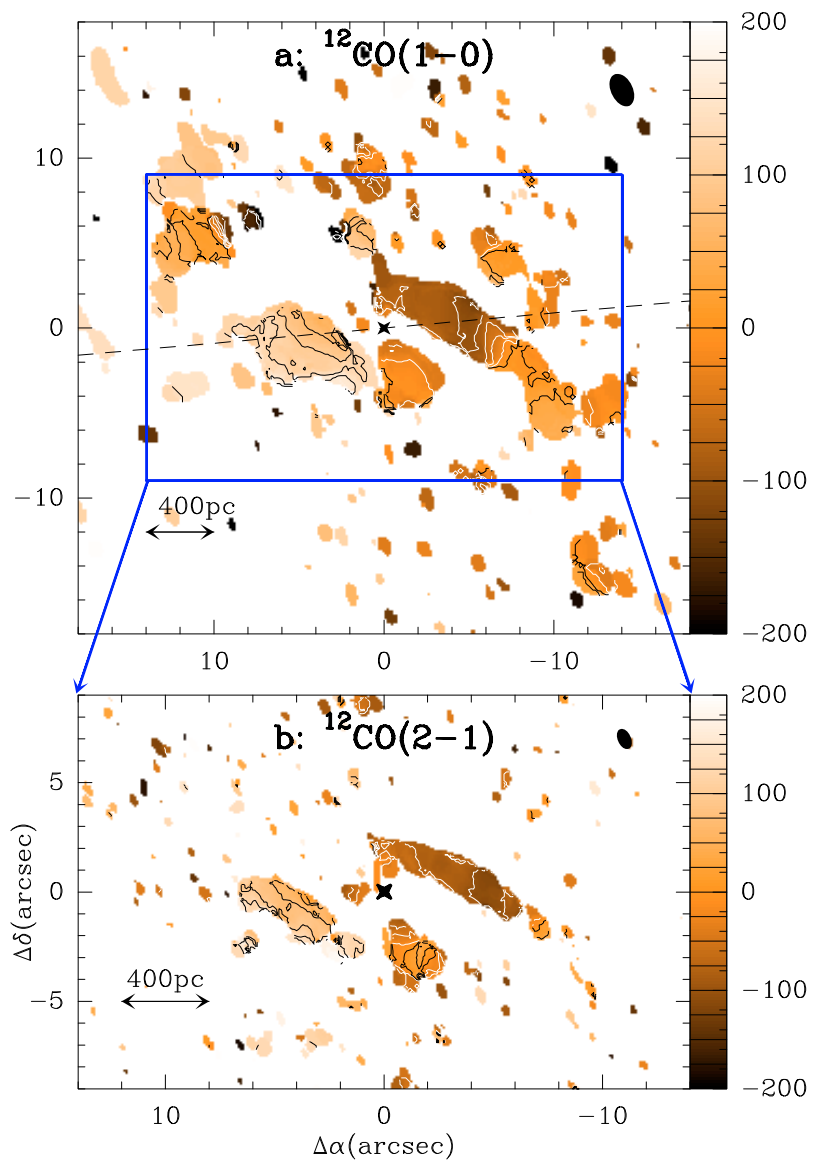

Fig. 9. $\mathrm{CO}(1-0)$ (upper panel) and $\mathrm{CO}(2-1)$ (lower panel) isovelocities contoured over false-color velocity maps. Velocities span the range $\left(-200 \mathrm{~km} \mathrm{~s}^{-1}, 200 \mathrm{~km} \mathrm{~s}^{-1}\right)$ in steps of $25 \mathrm{~km} \mathrm{~s}^{-1}$. Velocity scale is relative to $v_{\text {sys }}$. Isovelocities have been derived using a 4- $\sigma$ clipping on both data cubes. The AGN position is marked with a star. The position of the major axis at PA $=95^{\circ}$ is indicated in the upper panel by the dashed line. 

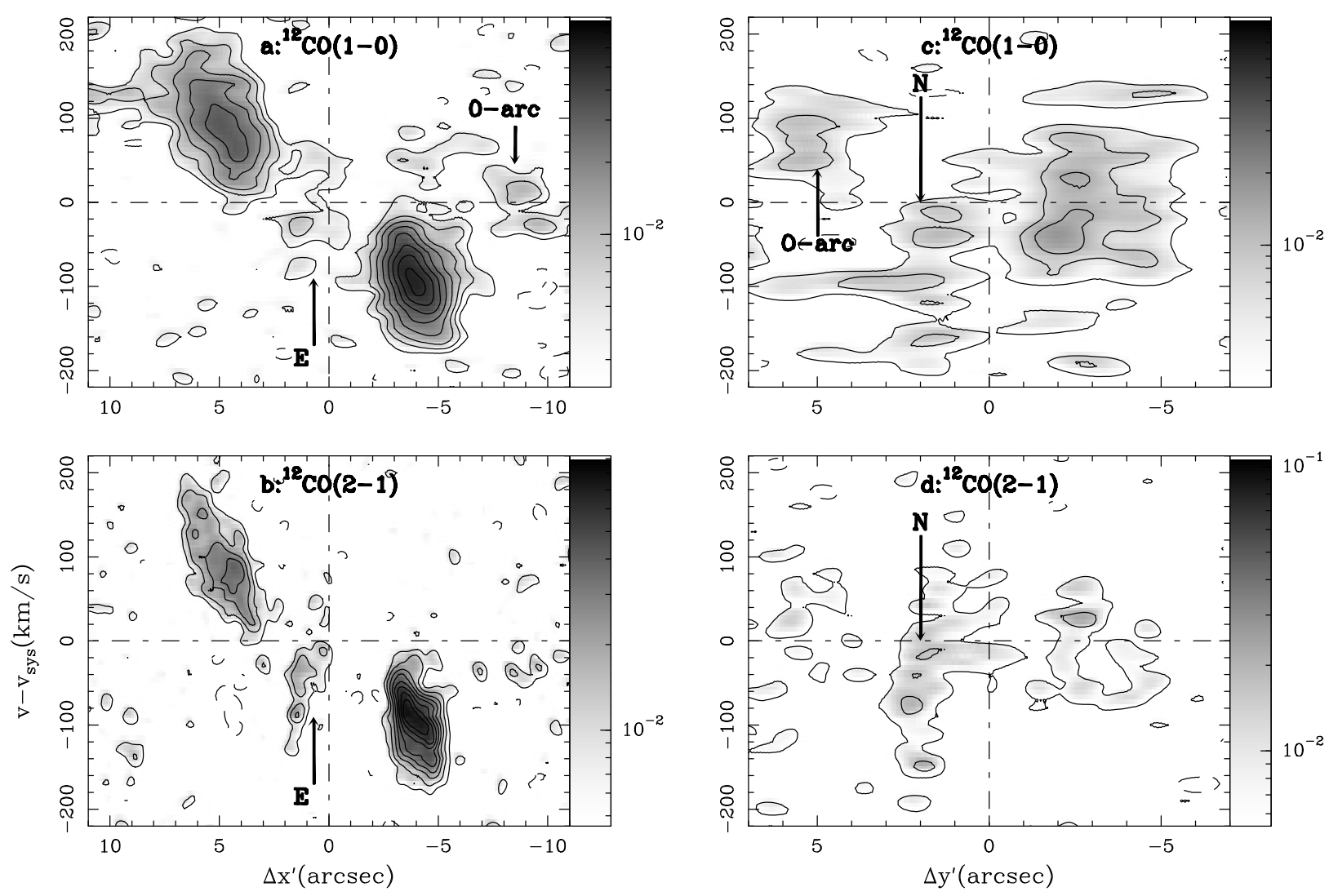

Fig. 10. a) Position-velocity diagram of $\mathrm{CO}(1-0)$ along the mayor axis of NGC $4579\left(\mathrm{PA}=95^{\circ}\right)$. Contour levels go from $-1.5 \sigma, 1.5 \sigma, 3 \sigma, 5 \sigma$, $8 \sigma$ to $24 \sigma$ in steps of $4 \sigma$ where the 1-sigma rms $\sigma=2.3 \mathrm{mJy} \mathrm{beam}^{-1}$. b) Same as a) but for $\mathrm{CO}(2-1)$ line with levels going from $-1.5 \sigma, 1.5 \sigma$, $3 \sigma, 5 \sigma, 7 \sigma$ to $15 \sigma$ in steps of $2 \sigma$ where the 1 -sigma rms $\sigma=4.6 \mathrm{mJy}_{\text {beam }}^{-1}$. c) Position-velocity diagram of $\mathrm{CO}(1-0)$ along the minor axis of NGC $4579\left(\mathrm{PA}=5^{\circ}\right)$. Contour levels are $-1.5 \sigma, 1.5 \sigma, 3 \sigma$ and $\left.5 \sigma . \mathbf{d}\right)$ Same as c) but for the $\mathrm{CO}(2-1)$ line. Contour levels are $-1.5 \sigma, 1.5 \sigma$ and $3 \sigma$. In all panels velocities are relative to $v_{\text {sys }}$ and both $\Delta x^{\prime}$ and $\Delta y^{\prime}$ offsets along the major and minor axis, respectively, are relative to the AGN locus. The position of the $\mathrm{N}$ and $\mathrm{E}$ complexes and that of the $\mathrm{O}$-arc are highlighted.

\subsection{Streaming motions}

The kinematics of molecular gas in the central $r \sim 2 \mathrm{kpc}$ of NGC 4579 reveal the presence of non-circular motions that modulate the overall rotation pattern of the disk described in Sect. 6.1. Deviations are detected with uneven strength over the inner spiral arms ( $\mathrm{N}$-arm and $\mathrm{S}$-arm), the outer arc (O-arc), and, most noticeably, over the central disk, as described below:

- The central disk: the radial velocities of the gas in the central disk-like structure deviate by up to $>100 \mathrm{~km} \mathrm{~s}^{-1}$ from the expected pattern of circular rotation. $\mathrm{CO}$ emission from the E complex, mostly prominent in the $2-1$ line, is detected at negative radial velocities $\left(\sim-70 \mathrm{~km} \mathrm{~s}^{-1}\right)$ that are forbidden by circular rotation at this location of the "disk" (see Figs. 8 and 10). If the gas flows inside the plane, this would imply that the gas at $\mathrm{E}$ is counter-rotating at a deprojected speed of $v \sim 120 \mathrm{~km} \mathrm{~s}^{-1}$. Complex N, which lies close to the kinematic minor axis of the galaxy, also shows non-circular motions: instead of a zero average velocity, we measured $\sim-50 \mathrm{~km} \mathrm{~s}^{-1}$ (see Figs. 8 and 10); this can be interpreted as gas moving outward within the plane. The CO kinematics observed in complexes $\mathrm{N}^{\prime}$ and $\mathrm{E}^{\prime}$ indicate instead positive radial velocities for the gas: $\sim+75 \mathrm{~km} \mathrm{~s}^{-1}$ at $\mathrm{N}^{\prime}$ and $\sim+25 \mathrm{~km} \mathrm{~s}^{-1}$ at $\mathrm{E}^{\prime}$. Altogether, the radial velocities measured along a line intersecting complexes $\mathrm{N}, \mathrm{N}^{\prime}, \mathrm{E}^{\prime}$ and $\mathrm{E}$ show a gradient that is reversed with respect to that expected if circular rotation prevailed.
- The inner spiral arms: the isovelocity maps of Fig. 9 reveal a steep transverse velocity gradient across the S-arm. An inspection of the major axis $p-v$ diagrams of Fig. 10 indicates that the gas is strongly slowed down to zero rotation velocities when it encounters the S-arm at $\Delta x^{\prime} \sim+3^{\prime \prime}$. Downstream the $\mathrm{S}$-arm (at $\left.\Delta x^{\prime} \sim+7^{\prime \prime}\right)$, gas is accelerated: rotation velocities $v_{\text {rot }}=\left(v-v_{\text {sys }}\right) / \sin (i) \sim 170 / \sin (36) \sim 290 \mathrm{~km} \mathrm{~s}^{-1}$ are reached when the gas leaves the arm. A similar but more shallow transverse gradient is detected across the $\mathrm{N}$-arm.

- The outer arc: Fig. 9 shows that the O-arc is also associated with peculiar motions. The radial velocities measured in adjacent regions of the $\mathrm{N}$-arm and $\mathrm{O}$-arc can differ by up to $150 \mathrm{~km} \mathrm{~s}^{-1}$. This is illustrated in Fig. 10 which shows the $p-v$ plots along the major and minor axis of the galaxy.

The streaming motions detected over the inner spiral arms are in agreement with the expected kinematic signatures of the stellar bar on the gas flow within the ILR region, and thus, well inside corotation of the bar (e.g., see Canzian 1993). However, the kinematic differences between the $\mathrm{N}$-arm and the $\mathrm{S}$-arm point to the possible influence of a superposed lopsided instability that could also be at play in the central disk-like component.

\subsection{An outflow of molecular gas in NGC 4579?}

Alternatively, the overall morphology and velocity pattern of the central disk could be qualitatively explained by a scenario where the gas is flowing away from the nucleus in extra-planar 

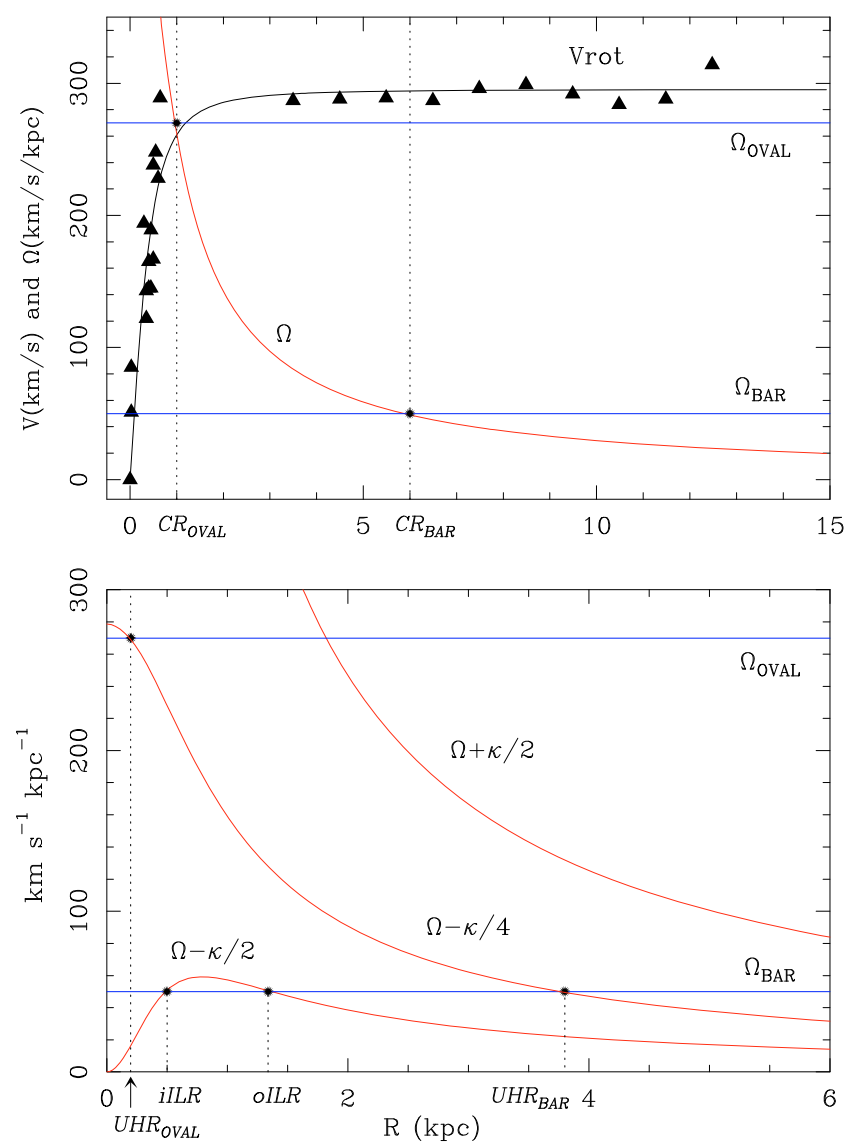

Fig. 11. a) (Upper panel) Rotation curve $v_{\text {rot }}$ and angular speed curve $\Omega$ fitted from the $\mathrm{CO}$ and $\mathrm{HI}$ data of NGC 4579 (triangles are data points). A value for the bar pattern speed $\Omega_{\mathrm{BAR}} \sim 50 \mathrm{~km} \mathrm{~s}^{-1} \mathrm{kpc}^{-1}$ puts the corotation radius of the bar at $r_{\mathrm{CR}} \sim 6 \mathrm{kpc}$. Similarly, a corresponding pattern speed value of $\Omega_{\mathrm{OVAL}} \sim 270 \mathrm{~km} \mathrm{~s}^{-1} \mathrm{kpc}^{-1}$ puts the corotation radius of the oval at $\sim 1 \mathrm{kpc}$. b) (Lower panel) Frequency curves $\Omega \pm \kappa / 2$ and $\Omega-\kappa / 4$. If $\Omega_{\mathrm{BAR}} \sim 50 \mathrm{~km} \mathrm{~s}^{-1} \mathrm{kpc}^{-1}$, the ultra harmonic resonance (UHR) of the bar lies at $r_{\mathrm{UHR}} \sim 3.8 \mathrm{kpc}$. We estimate the existence of an extended ILR region with two ILRs lying at $r_{\text {iILR }} \sim 500 \mathrm{pc}$ (inner ILR $=$ iILR) and $r_{\text {oILR }} \sim 1.3 \mathrm{kpc}$ (outer ILR $=$ oILR), respectively. With $\Omega_{\text {OVAL }} \sim 270 \mathrm{~km} \mathrm{~s}^{-1} \mathrm{kpc}^{-1}$ the UHR of the oval is at $\sim 200 \mathrm{pc}$.

motions, as mentioned by GB05. The [OIII] line and UV continuum maps obtained with HST can be used to tentatively identify a one-sided fan-shaped morphology in the emission coming from the central $r \sim 100$ pc of NGC 4579 (Pogge et al. 2000; Contini 2004), suggestive of an AGN-powered outflow of hot gas. The morphology of the neutral gas disk at $r \leq 150 \mathrm{pc}$, evidenced by the $V-I$ color image of the galaxy, would indicate that the gas is piling up in a shell. The shell could be a signature of gas entrainment by the outflow (see Fig. 8). The expanding shell scenario could explain the kinematics of the $\mathrm{N}$ and $\mathrm{E}$ complexes as a signature of out-of-the-plane motions projected along the line of sight towards the observer. The positive radial velocities measured towards complexes $\mathrm{N}^{\prime}$ and $\mathrm{E}^{\prime}$ can be explained if out of the plane motions are projected away from the observer. In this scenario AGN feeding would be presently quenched on these scales.

The outflow scenario for NGC 4579 is not flawless, however. Firstly, based on the current data, the putative outflow of hot gas in NGC 4579 does not have the mass and energies typically required to produce a corresponding outflow in the neutral gas (e.g., see the case of NGC 4528 discussed by Krause et al. 2007). Furthermore, in NGC 4579 there is no evidence of a radio jet, which should be the driving agent of the outflow. Finally, there is no information on the kinematics of the ionized gas that may be indicative of outflow motions. On the whole, the outflow scenario can be considered as merely speculative.

\section{Comparison with other tracers}

In this section we compare the CO maps of NGC 4579 with the distribution shown by other tracers of the ISM and with the stellar structure of the galaxy. This information is used to estimate the location of the resonances in NGC 4579, a key to the interpretation of the gas-flow in the disk of the galaxy.

\subsection{The large-scale bar}

The $2.2 \mu \mathrm{m} K$-band image of NGC 4579 published by Knapen et al. (2003) shows a prominent stellar bar in the disk. The bar is oriented along $\mathrm{PA}_{\mathrm{bar}} \sim 56 \pm 5^{\circ}$ (measured east from north in the plane of the sky). Once deprojected onto the plane of the galaxy (assuming $\mathrm{PA}=95^{\circ}$ and $i=36^{\circ}$ ), the position angle of the bar $\left(\mathrm{PA}_{\mathrm{bar}}^{\prime}\right)$ measured from the major axis becomes: $\tan \left(\mathrm{PA}_{\mathrm{bar}}^{\prime}\right)=$ $\tan \left(\mathrm{PA}-\mathrm{PA}_{\mathrm{bar}}\right) / \cos (i)$, which implies $\mathrm{PA}_{\mathrm{bar}}^{\prime} \sim 45^{\circ}$, as shown in Fig. 12. An isophotal analysis made on the $K$-band image of the galaxy gives an approximate diameter-size for the bar of $D \sim$ 10-12 kpc. This coincides with the value derived from a similar analysis of the $3.6 \mu \mathrm{m}$ Spitzer/IRAC image shown as white contours in the upper panel of Fig. 14. This bar size is compatible within the errors with the value calculated from the Fourier decomposition of the potential, which favors a slightly larger bar $(D \geq 14 \mathrm{kpc})$, as discussed in Sect. 8.2.1.

In the following, we will suppose that the corotation resonance lies at the end of the bar: $r_{\mathrm{CR}} \sim 6 \pm 1 \mathrm{kpc}$, i.e., allowing for a $20 \%$ uncertainty in $r_{\mathrm{CR}}$. This is a plausible scenario for the well-developed although relatively weak bar of NGC 4579 (see Sect. 8). An estimate of the bar pattern speed $\left(\Omega_{\mathrm{BAR}}\right)$ is thus obtained using the $\Omega$ curve derived from $\mathrm{CO}$ if we rely on the epicyclic approximation; this yields $\Omega_{\mathrm{BAR}} \sim 50 \pm$ $10 \mathrm{~km} \mathrm{~s}^{-1} \mathrm{kpc}^{-1}$ (see Fig. 11). The two ILRs lie approximately at $r \sim 500 \mathrm{pc}$ (inner ILR $=\mathrm{iILR})$ and $r \sim 1.3 \mathrm{kpc}$ (outer ILR $=$ oILR) for $\Omega_{\mathrm{BAR}} \sim 50 \mathrm{~km} \mathrm{~s}^{-1} \mathrm{kpc}^{-1}$ (Fig. 11). This value of $\Omega_{\mathrm{BAR}}$ allows the bar to have an extended ILR region, a requirement imposed by the observed distribution and kinematics of molecular gas in the central $r \sim 2 \mathrm{kpc}$ disk. In the context of this approximation we can put an upper limit to the pattern speed of the bar of NGC 4579: $\Omega_{\mathrm{BAR}} \sim 60 \mathrm{~km} \mathrm{~s}^{-1} \mathrm{kpc}^{-1}$; this value of $\Omega_{\text {BAR }}$ would impose the existence of a single ILR at $\sim 800 \mathrm{pc}$, a scenario incompatible with observations, which require the existence of two ILRs. With $\Omega_{\mathrm{BAR}} \sim 50 \mathrm{~km} \mathrm{~s}^{-1} \mathrm{kpc}^{-1}$, the O-arc would correspond to the oILR, a resonance which is currently being depopulated due to gas inflow down to a region inside the iILR of the bar (see discussion in Sect. 8.2). The latter explains the molecular gas concentration in the inner spiral arms $(\mathrm{N}-a r m$ and $\mathrm{S}$-arm), formed as a mixture of $x_{2}$ and $x_{1}$ precessing orbits. On its way to the nucleus, molecular gas is forming stars in the inner spiral arms, as evidenced by the detected $\mathrm{H} \alpha$ emission, more intense in the case of the $\mathrm{N}$-arm (see Fig. 13), as discussed in Sect. 7.4.

\subsection{The nuclear oval and nuclear UV ring}

The $K$-band image of NGC 4579 shows evidence of a previously unnoticed weak oval distortion present in the inner $r \sim 150 \mathrm{pc}$ (Fig. 15). This nested structure was not detected in the $I$-band 


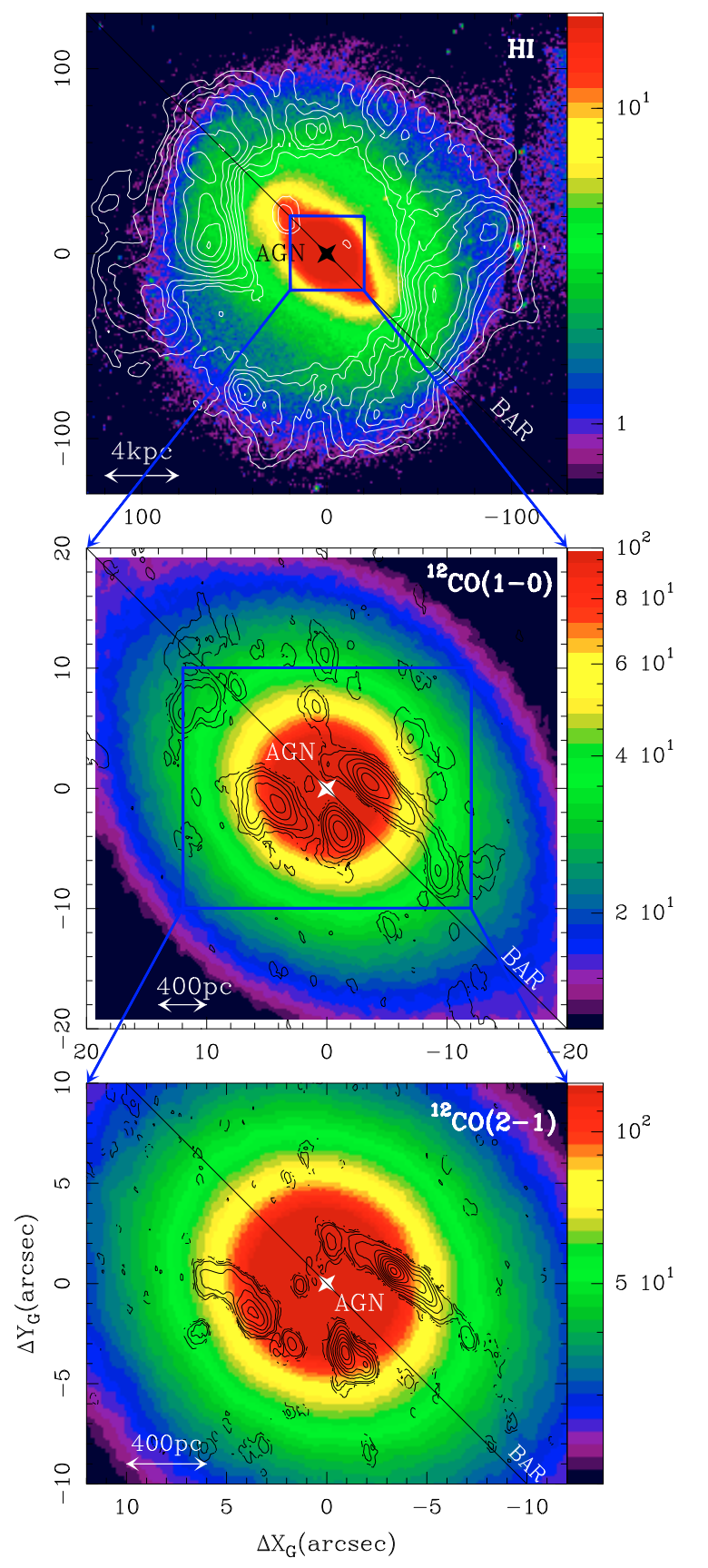

Fig. 12. a) (Upper panel) We overlay the HI intensity map obtained with the VLA (in contours from 0.04 to 0.28 in steps of $0.03 \mathrm{Jy} \mathrm{km} \mathrm{s}^{-1}$ beam ${ }^{-1}$ ) on the $K$-band WHT image (color scale) obtained for the nucleus of NGC 4579; both images have been deprojected onto the galaxy plane. Units on $X / Y$ axes $\left(\Delta X_{G} / \Delta Y_{G}\right)$ correspond to arcsec offsets along the major/minor axes with respect to the AGN. The orientation of the large-scale bar - BAR - is shown. b) (Middle panel) Same as a) but we overlaid the $\mathrm{CO}(1-0) \mathrm{PdBI}$ intensity map (contour levels $=0.2,0.5,1,1.7,2.6,3.7$ and $5 \mathrm{Jy} \mathrm{km} \mathrm{s}^{-1}$ beam $\left.{ }^{-1}\right)$. c) (Lower panel) Same as a) but for the $\mathrm{CO}(2-1) \mathrm{PdBI}$ intensity map (contour levels $=0.3,0.6,1$ to 9 in steps of $1 \mathrm{Jy} \mathrm{km} \mathrm{s}^{-1}$ beam $^{-1}$ ).

HST image originally used in the first gravity torque analysis of NGC 4579 (GB05). The oval is oriented along $\mathrm{PA}_{\text {oval }}^{\prime} \sim 70^{\circ}$ (measured from $+X$ axis in the plane of the galaxy), and therefore is not aligned with the large-scale bar. Misalignment can be taken as evidence of decoupling of the nested structure. The reality of this weak distortion is confirmed by the analysis of

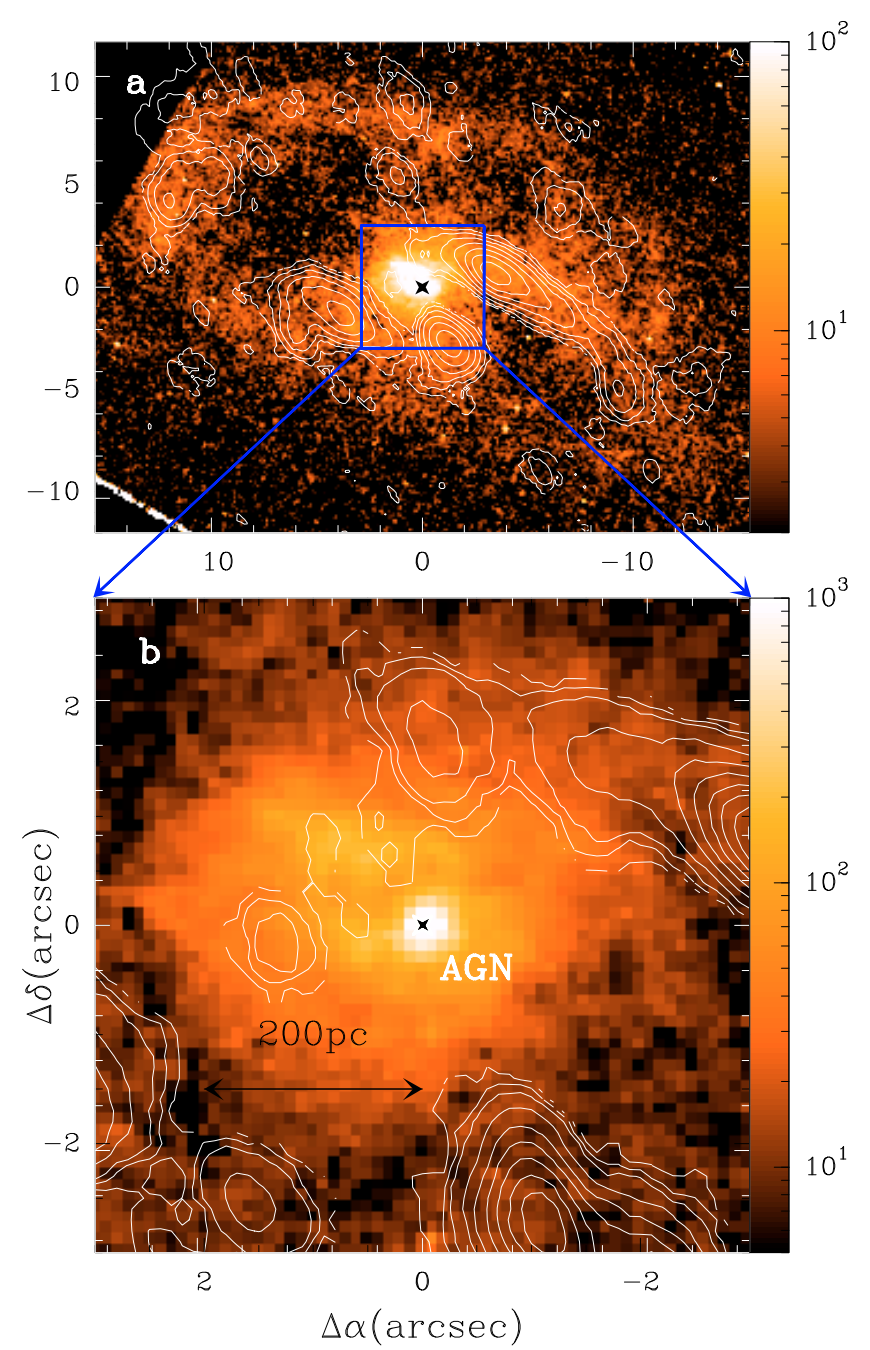

Fig. 13. Same contours as in Fig. 7 overlaid on the $\mathrm{H} \alpha$ image from HST (color scale) observed in the nucleus of NGC 4579.

the Fourier decomposition of the potential described in Sect. 8. The lower panel of Fig. 15 shows a zoomed view of the central $r \sim 300$ pc-region. The oval appears to be roughly cospatial with an off-centered ellipsoidal ring detected in the HST UV image of the galaxy, first discussed by Contini (2004). The UV ring has an average radius $r \sim 150-200 \mathrm{pc}$ and has been recently described by Comerón et al. (2008) as an ultra-compact nuclear ring.

The nuclear UV ring can be interpreted as a resonance of the oval distortion. The best guess assigns the ultra harmonic resonance (UHR) of the oval to the UV ring: if the pattern speed of the oval is $\Omega_{\text {OVAL }} \sim 270 \pm 10 \mathrm{~km} \mathrm{~s}^{-1} \mathrm{kpc}^{-1}$, the UHR of the oval should lie at $\sim 200 \mathrm{pc}$ and its corotation at $\sim 1 \mathrm{kpc}$ (Fig. 11). With this choice we can explain the decoupling of the oval with respect to the large-scale bar, as the corotation of the oval would be close to the oILR of the large-scale bar. This resonance overlap is a prerequisite to the decoupling of nuclear bars/ovals (e.g., García-Burillo et al. 1998; Hunt et al. 2008). The putative oval distortion is admittedly weak; nevertheless, because the Fourier decomposition points to such a feature, it neatly sets the stage for the resonances (e.g., nuclear UV ring) seen at other wavelengths.

As discussed in Sect. 8.2, the oval perturbation could favor gas fueling to the AGN at present. In particular, the decoupling scenario would explain the depopulation of the oILR of the large-scale bar, under the combined action of the bar and the nuclear oval. A similar time sequence is nicely illustrated by the 

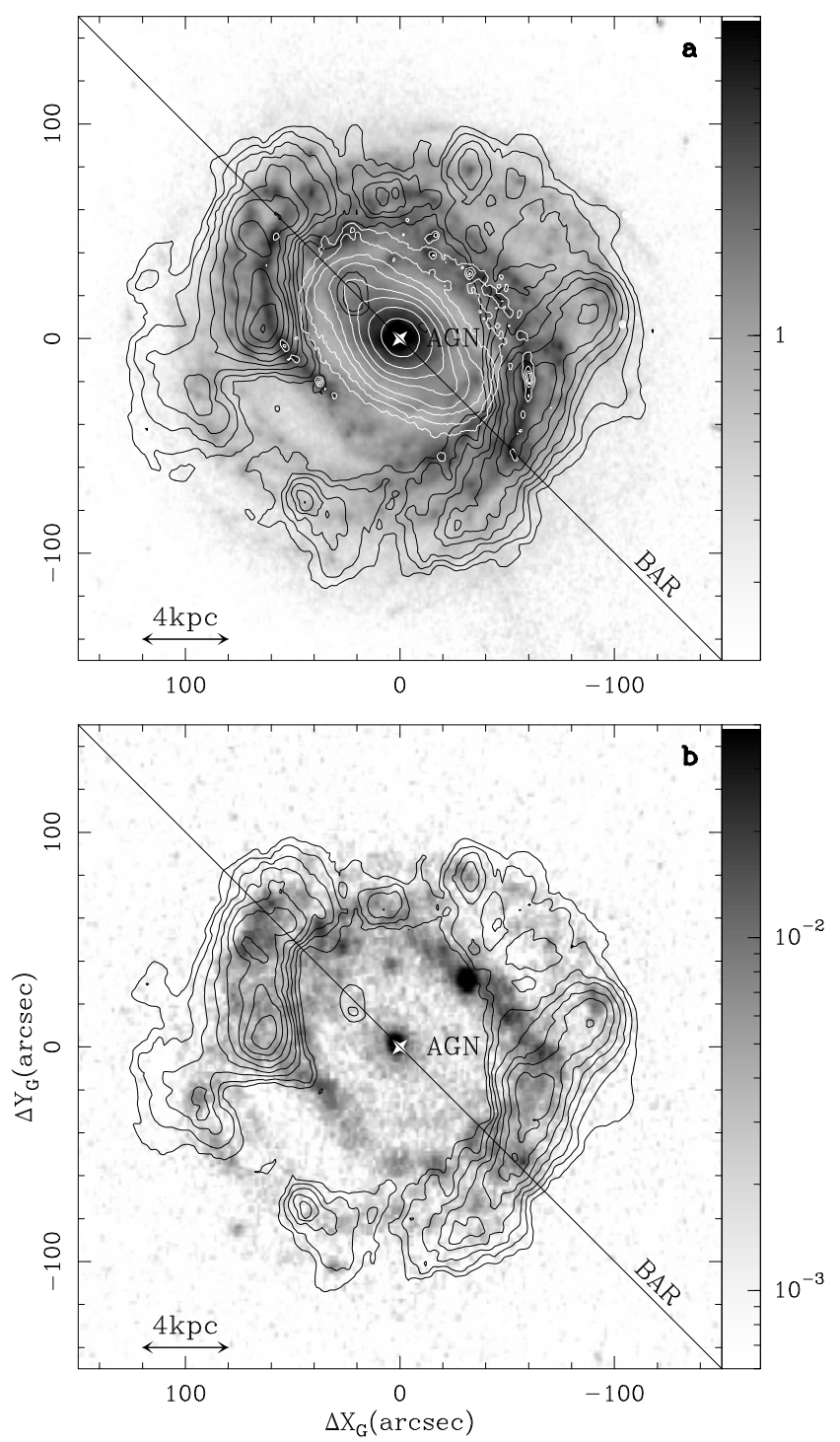

Fig. 14. a) (Upper panel) Superposition of the HI intensity map (black contours), the $3.6 \mu \mathrm{m}$ Spitzer/IRAC image in contour levels 1.1, 1.3, 1.6, 2, 3, 4, 6 and $11 \mathrm{MJy} / \mathrm{sr}$ (white contours) and the $8 \mu \mathrm{m}$ Spitzer/IRAC image (grey scale). All images have been deprojected onto the galaxy plane. b) (Lower panel) Same as a) but HI intensity map (in contours) superposed on the FUV GALEX image (grey scale).

numerical simulations adapted to follow the nuclear bar decoupling in the double-barred galaxy NGC 2782, discussed by Hunt et al. (2008).

\subsection{The gas distribution in the outer disk $(r>4 \mathrm{kpc})$}

The $21 \mathrm{~cm} \mathrm{HI} \mathrm{map} \mathrm{of} \mathrm{NGC} 4579$ gives a sharp view of the gas distribution in the outer disk of the galaxy (from $r \sim 4$ to $15 \mathrm{kpc}$ ). Figure $12 \mathrm{a}$ shows the HI map superimposed on the $K$-band deprojected image. Similarly, Fig. 14 shows the HI map superimposed on the Spitzer/IRAC 3.6, $8.0 \mu \mathrm{m}$ images (top panel), and the FUV GALEX images (bottom). The $8 \mu \mathrm{m}$ Spitzer/IRAC and the FUV GALEX images provide a detailed picture of the star formation in the disk. For an Sb galaxy such as NGC 4579, $>70 \%$ of the total emission at $8 \mu \mathrm{m}$ is of non-stellar origin and is produced by warm dust heated by star formation activity (e.g., Pahre et al. 2004). The HI distribution in the disk shows a central depression in the inner $r \sim 4 \mathrm{kpc}$, mimicking a ring. The

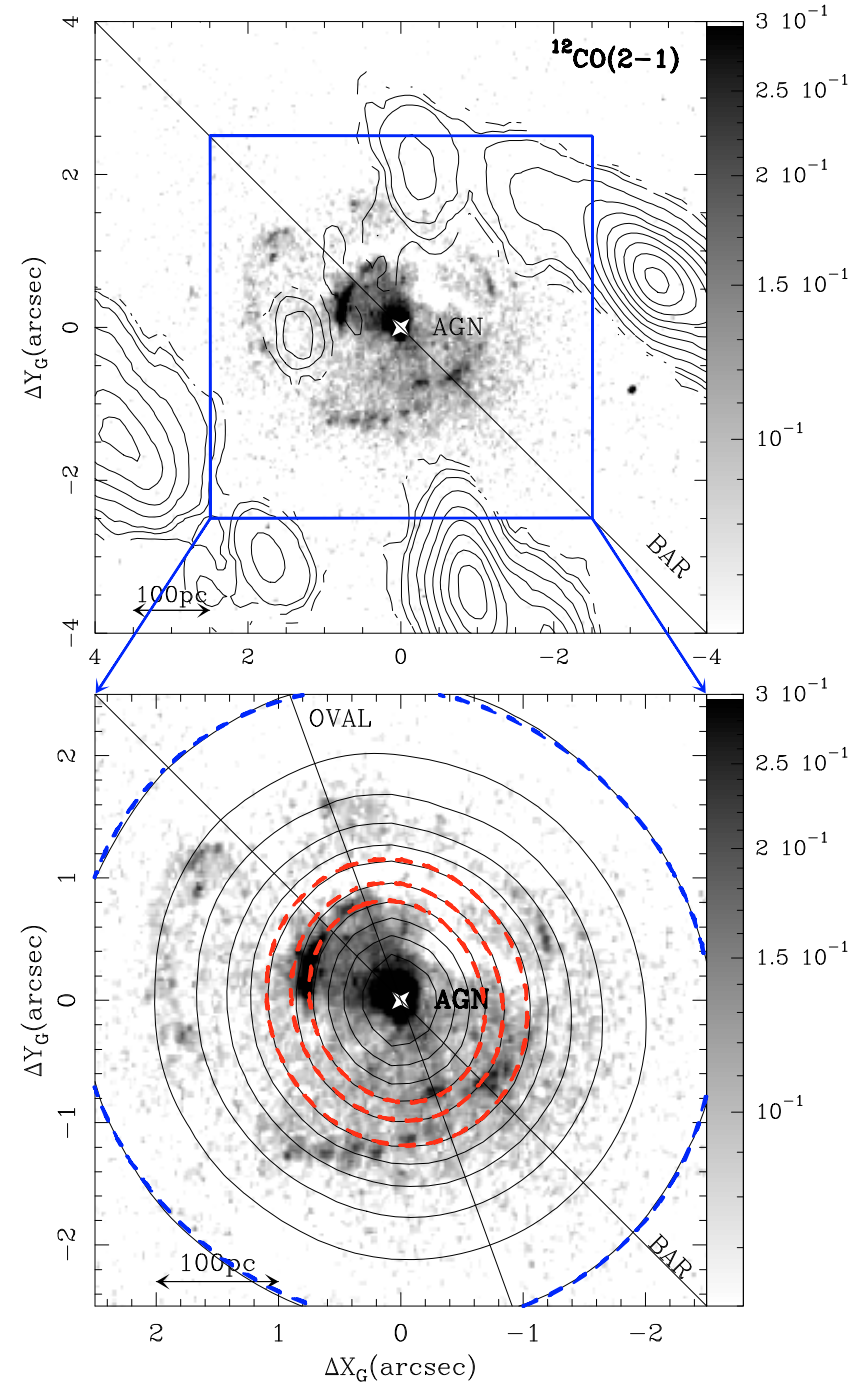

Fig. 15. a) (Upper panel) The ${ }^{12} \mathrm{CO}(2-1)$ integrated intensity map obtained with the PdBI (same contours as in Fig. 7) is overlaid on the UV image of the central region of NGC 4579 obtained with the ACS/HRC in the $F 330 W$ band (in grey scale). b) (Lower panel) The $K$-band contours are overlaid on the UV-image of the galaxy. To highlight the orientation of the bar and the oval, we show three ellipses fitted to the nuclear oval in the inner $r<100 \mathrm{pc}$ (thick dashed contours); the orientation of the isophotes changes towards the outer region, as shown by the fitted ellipse at $r \sim 300 \mathrm{pc}$ (thick dashed contour). All images have been deprojected onto the galaxy plane.

HI pseudo-ring is partly incomplete and has an average diameter of $\sim 12 \mathrm{kpc}$. At close sight, the HI pseudo-ring appears to be the superposition of two winding spiral arms. The inner segments of the spiral arms are better identified in the $8 \mu \mathrm{m}$ Spitzer/IRAC and the GALEX images; the ring-like structure seen in the $8 \mu \mathrm{m}$ and FUV emission neatly delineates the inner edge of the HI pseudoring. Further out, the arms unfold in the disk over $180^{\circ}$ in azimuth and end up forming the pseudo-ring detected in HI. Most remarkably, the spiral arms are not connected to the two bar ends located at $(r, \Phi)=\left(6 \mathrm{kpc}, 45^{\circ}\right)$ and $\left(6 \mathrm{kpc}, 225^{\circ}\right)$. Instead, the two-arm spiral structure starts at a different azimuth $\left(\sim 90^{\circ}\right.$ from the bar ends) and, also, at a significantly smaller radius in the disk: $(r, \Phi)=\left(4 \mathrm{kpc}, 135^{\circ}\right)$ and $\left(4 \mathrm{kpc}, 315^{\circ}\right)$.

On the whole, this geometry suggests that the bar and the spiral are decoupled and thus do not share a common pattern speed. It is accepted that spirals and bars can rotate at differing 
angular speeds in barred spiral galaxies, a scenario first advocated by Sellwood \& Sparke (1988) who contemplate that, under decoupling, outer spirals can rotate at lower $\Omega_{p}$. As recently shown by numerical simulations (e.g., Combes 2008, and references therein), decoupling is more likely in the case of weak bars, of which the NGC 4579's bar is a good example. As discussed in Sect. 8.2, this situation favors gas fueling to the central regions, as the corotation barrier imposed by the bar could be thus overcome. The morphology of the outer disk revealed by HI in NGC 4579 suggests that the neutral gas is currently piling up in an inner ring located well inside the corotation of the bar. This scenario is confirmed by the coincidence of the inner edge of the HI pseudo-ring with the predicted position of the UHR of the bar, around $r_{\mathrm{UHR}} \sim 3.8 \mathrm{kpc}$, as illustrated in Fig. 11 .

\subsection{The star formation rate in NGC 4579}

The star formation pattern in the inner disk $(r \leq 2 \mathrm{kpc})$ of NGC 4579 has been previously described by Pogge et al. (2000) and Contini (2004), based on HST optical and UV images (see also descriptions by GB05 and Comerón et al. 2008). Most of the $\mathrm{H} \alpha$ emission in the region $200 \mathrm{pc}<r<2 \mathrm{kpc}$ comes from a two-arm spiral structure with a geometry similar to that of the CO inner spiral arms, described in Sect. 4 (see Fig. 13). In addition, part of the $\mathrm{H} \alpha$ emission is related to the $\mathrm{CO}$ outer arc. Closer to the AGN ( $r<200 \mathrm{pc}$ ), the bulk of the $\mathrm{H} \alpha$ emission is seen to come from a slightly off-centered disk. At close sight, several subcomponents can be identified in this disk. There is a point-like nuclear source of $\sim 0.27^{\prime \prime}$-size, located at the dynamical center of the galaxy; the emission of this component has been interpreted as due to photo-ionization by the AGN (Maoz et al. 2005). Furthermore, the [OIII] line and UV maps reveal also a one-sided fan-shaped morphology in the emission in the inner $r \sim 100$ pc of NGC 4579 (Pogge et al. 2000; Contini 2004). On larger scales, the HST UV image of the galaxy, shows an offcentered ring at $r \sim 150-200$ pc (see Sect. 7.2 and Comerón et al. 2008). As described in Sect. 7.3, star formation in the outer disk, as traced by the UV continuum emission seen by GALEX, arises from a pseudo-ring characterized by a geometry similar to that of the HI pseudo-ring.

In order to quantify the amount of gas transformed into stars, we have used the HST H $\alpha$ and GALEX UV images described above to calculate the corresponding star formation rates (SFR) on different regions throughout the disk of the galaxy. SFR values are derived using the empirical formulae given by Kennicutt (1998). We first estimate the SFR in the inner disk; more precisely, we define $\mathrm{SFR}_{\text {inner }}$ as the integrated SFR inside the region $300 \mathrm{pc}<r<2 \mathrm{kpc}$. To correct for extinction, and thus have a more accurate evaluation of $\mathrm{SFR}_{\text {inner }}$, we have used the $\mathrm{CO}(1-0) \mathrm{PdBI}$ map to derive the $\mathrm{H}_{2}$ column density map, using the CO-to- $\mathrm{H}_{2}$ conversion factor of Solomon \& Barrett (1991): $X=N\left(\mathrm{H}_{2}\right) / I_{\mathrm{CO}}=2.3 \times 10^{20} \mathrm{~cm}^{-2} \mathrm{~K}^{-1} \mathrm{~km}^{-1} \mathrm{~s}$. The $A_{V}$ map is then derived using the commonly-used $N\left(\mathrm{H}_{2}\right)$-to- $A_{v}$ conversion factor of Bohlin et al. (1978): $N\left(\mathrm{H}_{2}\right) / A_{V}=10^{21} \mathrm{~cm}^{-2} \mathrm{mag}^{-1}$. Adopting a screen-geometry for the obscuring dust in front of the HII regions, we must scale the $A_{v}$ map by a factor $1 / 2$, if HII regions are assumed to lie in the mid-plane of the galaxy. This scaled extinction map, with $A_{v}$ typically ranging from $\sim 3$ to $\sim 15$, is used to derive $A_{\mathrm{H} \alpha}$, following Cardelli et al. (1989): $A_{\mathrm{H} \alpha}=0.828 \times A_{v}$. We derive $S F R_{\text {inner }} \sim 0.10 M_{\odot} \mathrm{yr}^{-1}$, confirming that the nuclear starburst of NGC 4579 is only moderate. In a second step, we use $\mathrm{SFR}_{\text {inner }}$ to calculate the SFR throughout the disk of the galaxy by scaling the integrated fluxes measured in the GALEX UV image at the corresponding radii. We define
$\mathrm{SFR}_{\text {outer }}$ as the SFR integrated inside the region $4 \mathrm{kpc}<r<$ $10 \mathrm{kpc}$, and derive $\mathrm{SFR}_{\text {outer }} \sim 1.30 M_{\odot} \mathrm{yr}^{-1}$. SFR $\mathrm{Stal}_{\text {tol }}$ is just the total SFR integrated to the limit of the GALEX image.

$\mathrm{SFR}_{\text {inner }}$ and $\mathrm{SFR}_{\text {outer }}$ contain the bulk of the total SFR of NGC 4579 estimated as SFR total $\sim 1.70 M_{\odot} \mathrm{yr}^{-1}$. In Sect. 8.2.3 these SFRs will be compared to the corresponding inflow rates driven by gravity torques in these regions, relevant to understand the overall gravity torque budget.

\section{Gravity torques}

\subsection{Methodology}

Firstly we have derived a good representation of the stellar potential for the disk of NGC 4579. To do so we have used the $K$-band image of the galaxy published by Knapen et al. (2003), assuming that this image is virtually free from dust extinction or stellar population biases. This working hypothesis better applies to the $K$-band image than to the $I$-band image originally used by GB05 to derive the gravity torque budget in NGC 4579. The $K$-band image has been deprojected according to the angles PA = $95^{\circ}$ and $i=36^{\circ}$, assumed in Sect. 1 . Figure 12 shows the overlay of the deprojected gas distribution as derived from the $\mathrm{CO}$ and HI maps with the deprojected $K$-band image of NGC 4579.

To reproduce the vertical mass distribution, the $K$-band image was convolved using an isothermal plane model of constant scale height, equal to $\sim 1 / 12$ th of the radial scale length of the image. We also adopted a constant mass-to-light (M/L) ratio, obtained by fitting the rotation curve to the $\mathrm{CO}$ and HI data. The potential - $\Phi(R, \theta)$ - was then calculated by a Fourier transform method and decomposed in the different $m$-modes as follows

$\Phi(R, \theta)=\Phi_{0}(R)+\sum_{m} \Phi_{m}(R) \cos \left(m \theta-\phi_{m}(R)\right)$

where $\Phi_{m}(R)$ and $\phi_{m}(R)$ are the amplitude and phase of the $m$-mode, respectively.

According to Combes \& Sanders (1981), the strength of the $m$-Fourier component is defined as: $Q_{m}(R)$ as

$Q_{m}(R)=m \Phi_{m} / R\left|F_{0}(R)\right|$.

Similarly, the strength of the total non-axisymmetric perturbation is obtained by

$Q_{T}(R)=\frac{F_{T}^{\max }(R)}{F_{0}(R)}=\frac{\frac{1}{R}\left(\frac{\partial \Phi(R, \theta)}{\partial \theta}\right)_{\max }}{\frac{\mathrm{d} \Phi_{0}(R)}{\mathrm{d} R}}$

where $F_{T}^{\max }(R)$ represents the maximum amplitude of the tangential force over all $\theta$ and $F_{0}(R)$ is the mean axisymmetric radial force. Figure 16 shows the description of the gravitational potential of NGC 4579 defined by the $Q_{i=1,2}, Q_{T}$, and $\phi_{i=1,2}$ curves, used in the discussion of Sect. 8.2.1.

The forces per unit mass $\left(F_{x}\right.$ and $\left.F_{y}\right)$ are calculated from the derivatives of $\Phi(R, \theta)$ at each pixel; the gravity torques per unit mass - $t(x, y)$ - can be then computed by

$t(x, y)=x F_{y}-y F_{x}$.

The sense of the circulation of the gas in the galaxy plane (counterclockwise in NGC 4579; see Sect. 6) defines whether the sign of $t(x, y)$ is positive (negative) if the torque accelerates (decelerates) locally the gas in the disk. Note that the specific torque field $t(x, y)$ does not depend on the present distribution of the gas. However, we use the $t(x, y)$ map to infer the angular momentum variations by assuming that the gas column density $(N(x, y))$ 

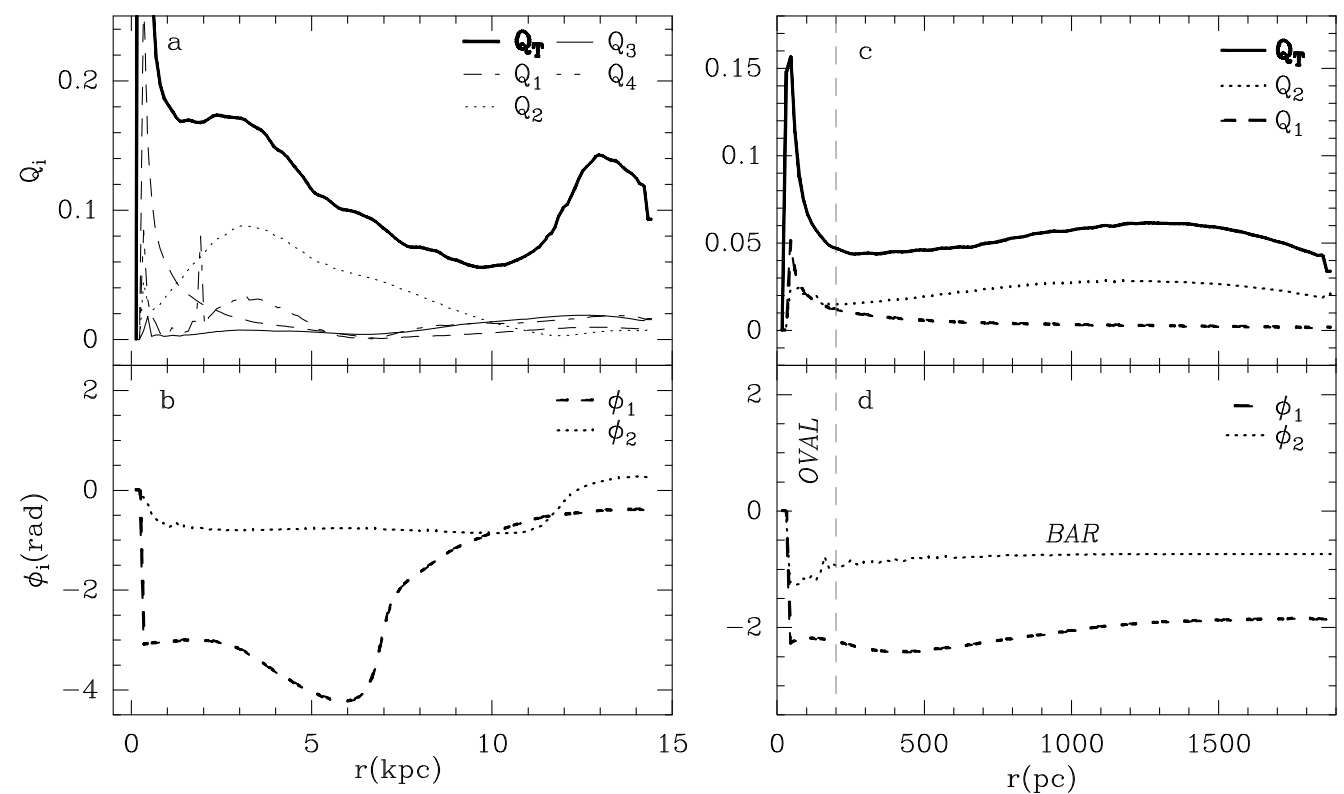

Fig. 16. Strength $\left(Q_{1}, Q_{2}\right.$ and $\left.Q_{T}\right)$ and phases $\left(\phi_{1}\right.$ and $\left.\phi_{2}\right)$ of $m=1$ and $m=2$ Fourier components of the stellar potential derived from the $K$-band WHT image inside $r \sim 15 \mathrm{kpc}$ (left panels: a), b)) and inside $r \sim 2 \mathrm{kpc}$ (right panels: c), d)). We highlight the radii where the OVAL and the BAR perturbations prevail at $r \leq 2 \mathrm{kpc}$. In addition, $Q_{3}$, and $Q_{4}$ values are plotted in panel a).

derived from the $\mathrm{CO}$ line maps (in the inner disk) and from the HI map (in the outer disk) is a good estimate of the probability of finding gas particles at this location at present. The torque field is then weighted by $N(x, y)$ at each pixel to derive the time derivative of the angular momentum surface density in the galaxy plane, $\mathrm{d} L_{s}(x, y) / \mathrm{d} t=N(x, y) \times t(x, y)$. Our approach is statistical since we do not follow particles along individual orbits, at best estimated through model fitting (e.g., see Boone et al. 2007), in order to derive angular momentum variations. Instead, we average over all orbits of gas particles at each position and account for the time spent by the gas clouds along all the possible orbit paths. Figure 18 shows the dimensionless version of the torque maps normalized by $[|N(x, y) \times t(x, y)|]_{\max }$, as derived from $\mathrm{CO}$ and HI. Sect. 8.2.2 provides a detailed description of these maps.

To estimate the net radial gas flow globally induced by the torque field in the galaxy disk, we have computed the torque per unit mass averaged over the azimuth $\theta$ as a function of radius $R$, using $N(x, y)$ as weighting function

$t(R)=\frac{\int_{\theta} N(x, y) \times\left(x F_{y}-y F_{x}\right)}{\int_{\theta} N(x, y)}$.

By definition, $t(R)$ is the azimuthally-averaged time derivative of the specific angular momentum $-L-$ of the gas, i.e., $t(R)=$ $\mathrm{d} L /\left.\mathrm{d} t\right|_{\theta}$. Similarly to the torque maps, the sign of $t(R)$, either positive or negative, defines whether the gas gains or loses angular momentum, respectively. Specifically, we estimate the AGN feeding efficiency by deriving the average fraction of the gas specific angular momentum transferred in one rotation $\left(T_{\text {rot }}\right)$ by the stellar potential, as a function of radius, i.e., by the dimensionless function $\Delta L / L$ defined as

$\frac{\Delta L}{L}=\left.\frac{\mathrm{d} L}{\mathrm{~d} t}\right|_{\theta} \times\left.\frac{1}{L}\right|_{\theta} \times T_{\text {rot }}=\frac{t(R)}{L_{\theta}} \times T_{\text {rot }}$

where $L_{\theta}$ is assumed to be well represented by its axisymmetric average, $L_{\theta}=R \times v_{\text {rot }}$. The absolute value of $L / \Delta L$ determines how much time (in $T_{\text {rot }}$ units) the stellar potential will need to transfer the entirety of the gas angular momentum. A low value of $\Delta L / L$ implies that the stellar potential is inefficient at present. The $\Delta L / L$ curves derived from the $\mathrm{CO}$ and $\mathrm{HI}$ maps of NGC 4579, shown in Fig. 19, are used in the discussion of Sect. 8.2.2.

Following the approach of GB05, we can estimate the gas mass inflow (-sign)/outflow ( + sign) rate driven by the stellar potential per unit length as a function of radius (in units of $M_{\odot} \mathrm{yr}^{-1} \mathrm{pc}^{-1}$ in Figs. 21a to 23a) according to

$\frac{\mathrm{d}^{2} M}{\mathrm{~d} R \mathrm{~d} t}=\left.\frac{\mathrm{d} L}{\mathrm{~d} t}\right|_{\theta} \times\left.\frac{1}{L}\right|_{\theta} \times 2 \pi R \times\left. N(x, y)\right|_{\theta}$

where $\left.N(x, y)\right|_{\theta}$ is the radial profile of $N(x, y)$ averaged over the azimuth for a radial binning $\Delta R$, derived from HI, for the outer disk, and from $\mathrm{CO}$, for the inner disk.

Similarly, the inflow/outflow rates integrated out to a certain radius $R$ can be derived as

$\frac{\mathrm{d} M}{\mathrm{~d} t}=\sum \frac{\mathrm{d}^{2} M}{\mathrm{~d} R \mathrm{~d} t} \times \Delta R$.

Figures $21 \mathrm{~b}$ to $23 \mathrm{~b}$ display these integrated rates in units of $M_{\odot} \mathrm{yr}^{-1}$. The mass transfer rates in NGC 4579 are discussed in Sect. 8.2.3.

\subsection{The gravity torque budget in NGC 4579}

\subsubsection{The stellar potential}

To derive the stellar potential $\Phi(R, \theta)$ used in the gravity torque calculation of NGC 4579, we have followed an adaptive approach. $\Phi(R, \theta)$ in the inner $r \sim 2 \mathrm{kpc}$ disk has been derived using a grid of $256 \times 256$ pixels with $0.15^{\prime \prime}$ pixel $^{-1}$ on the $K$-band image. This representation of $\Phi(R, \theta)$ is the best adapted to derive the torque field on $\mathrm{CO}$. On the other hand, a spatially smoothed version of the NIR image, with $256 \times 256$ pixels and $1.15^{\prime \prime} \mathrm{pixel}^{-1}$, has been used to calculate $\Phi(R, \theta)$ in the outer disk up to $r \sim 15 \mathrm{kpc}$. The ensuing torque field on $\mathrm{HI}$ is derived from this representation of the stellar potential. 


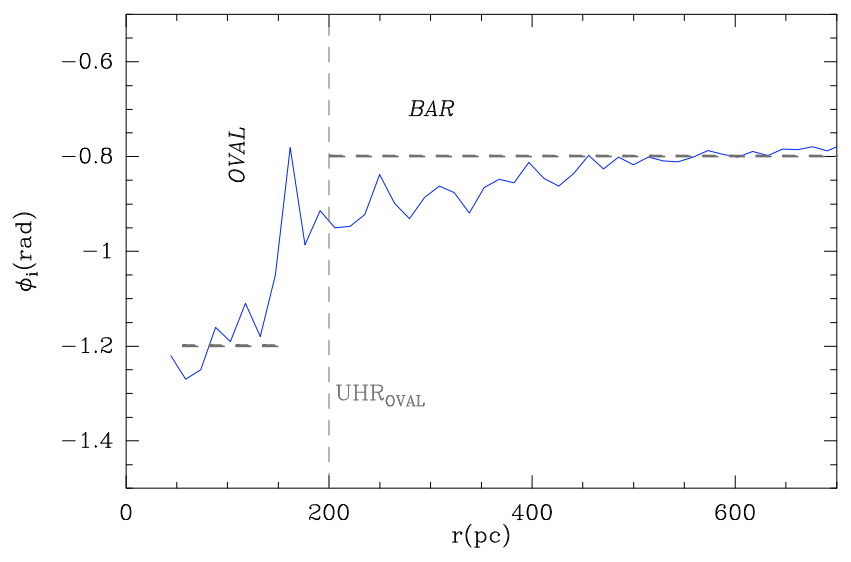

Fig. 17. Same as Fig. 16d but zooming on the inner $r \sim 700 \mathrm{pc}$ disk of the galaxy. We illustrate the drift in the phase of the $m=2$ mode $\left(\phi_{2}\right)$ from the value characterizing the bar phase $\left(\phi_{2}[\mathrm{BAR}] \sim-0.8 ; \mathrm{PA}=\right.$ $\left.45^{\circ}\right)$, to the value of the nuclear oval $\left(\phi_{2}[\mathrm{OVAL}] \sim-1.2 ; \mathrm{PA}=70^{\circ}\right)$. The average phase of the bar has been fitted over $200 \mathrm{pc}<r<6 \mathrm{kpc}$; the average phase of the oval has been fitted over the inner $r<150 \mathrm{pc}$, after blocking the central $r<50 \mathrm{pc}$ region.

Figure 16 shows the $Q$ values derived as a function of radius. The large-scale bar $(m=2)$ is clearly detected in the smoothed version of $\Phi(R, \theta)$ (Figs. 16a and b). The area of influence of the bar, determined by the dominance of the $m=2$ mode over other secondary modes extends up to $r \sim 7-8 \mathrm{kpc}$ in the disk. For $r \geq 8 \mathrm{kpc}, Q_{1}+Q_{3}+Q_{4} \geq Q_{2}$, an indication that the bar mode is not dominant on these scales of the outer disk. This puts an upper limit to the size of the bar $(r<7-8 \mathrm{kpc})$. As discussed in Sect. 7.1, the isophotal analysis of the NIR images of the galaxy gives a smaller size for the bar: $r \sim 5-6 \mathrm{kpc}$. The finally adopted value for $r_{\mathrm{CR}}=6 \pm 1 \mathrm{kpc}$ is a reasonable compromise between the two determinations above and it accounts for the overall gas response in the disk.

On the whole, the bar is moderately strong: the $Q_{2}$ strength of the bar is maximum at $r \sim 3 \mathrm{kpc}$ where it reaches a value of $\sim 0.1$. Moreover, the phase of the bar $\phi_{2}$ stays noticeably constant at a value $\sim-0.8$ radians, i.e., at an angle $\sim 45^{\circ}$, measured counterclockwise from $+X$-axis in Fig. 12. This also confirms the value of $\mathrm{PA}_{\mathrm{bar}}^{\prime}$ derived from the isophotal analysis of Sect. 7.1. The inner representation of $\Phi(R, \theta)$ also reveals the bar at $r<$ $2 \mathrm{kpc}$ (Figs. 16c and d), with a similarly constant phase and, not surprisingly, with an average lower strength, $Q_{2} \sim 0.02-0.03$ (at these radii we are inside the peak of $Q_{2}$ ). Of particular note, the high-resolution version of $\Phi(R, \theta)$ unveils the oval distortion with a size and an orientation similar to those derived in Sect. 7.2. This is illustrated in Fig. 17, which shows how the phase of the $m=2$ mode changes in the inner $r \sim 700 \mathrm{pc}$ disk of the galaxy. The presence of a misaligned oval is betrayed by a significant $\sim 25^{\circ}$ change in the phase of the $m=2$ mode. $\phi_{2}$ gradually shifts from the large-scale bar orientation, $\sim-0.8$ radians for $200 \mathrm{pc}<r<7 \mathrm{kpc}\left(\sim 45^{\circ}\right)$, to $\sim-1.2$ radians $\left(\sim 70^{\circ}\right)$ at $r \sim 100 \mathrm{pc}$.

There are discrepancies, most likely attributable to dust extinction, between the stellar potential obtained from the $K$-band image of NGC 4579, and the representation of $\Phi(R, \theta)$ obtained by GB05 from the $I$-band HST image of the galaxy. Firstly, the strong $m=1$ mode detected in the $I$-band image is much weaker in the new evaluation of $\Phi(R, \theta)$. As illustrated in Fig. 7 of GB05, the $m=1$ mode seemed to overtake the potential strength of the bar at $r<500$ pc: $Q_{1}$ was seen to vary between $\sim 0.02$ and $\sim 0.10$, while $Q_{2} \sim 0.02$. This is in contrast to the new result shown in

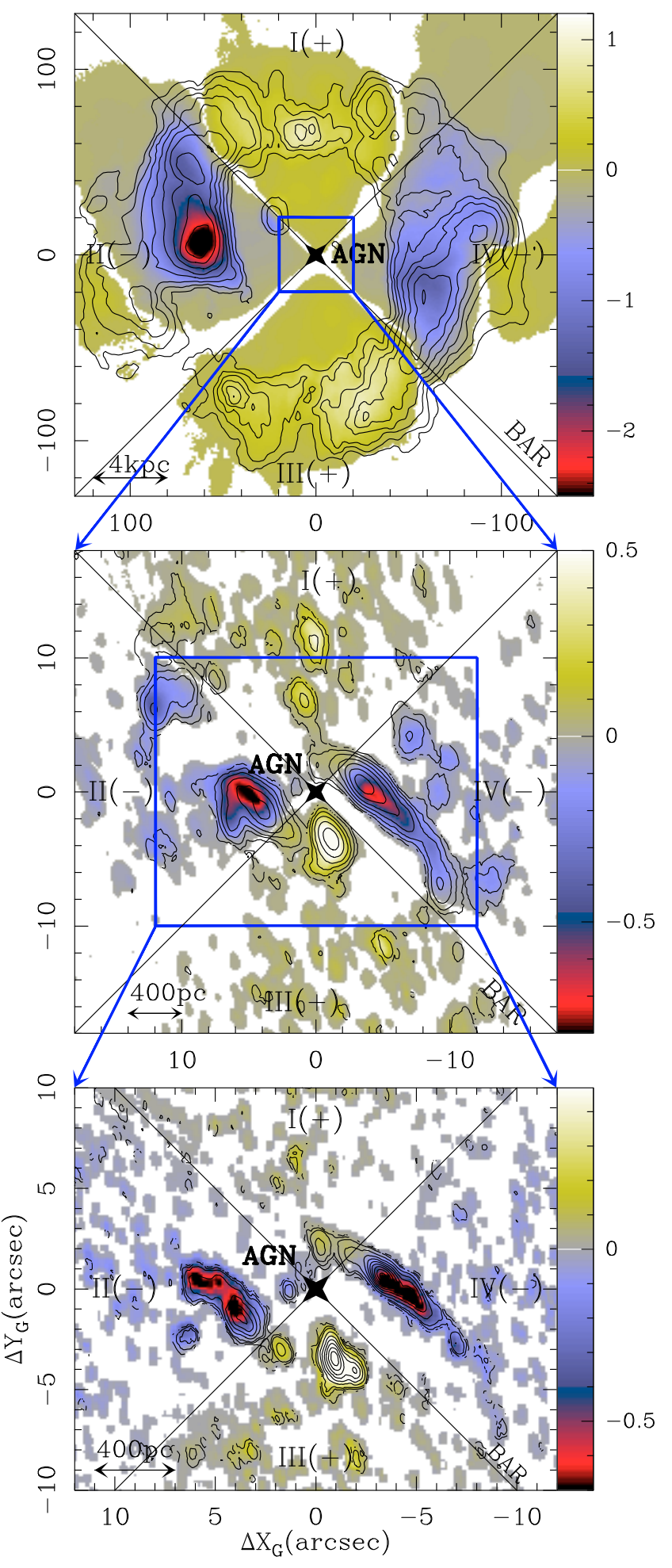

Fig. 18. a) (Upper panel) We overlay the HI contours with the map of the (dimensionless) effective angular momentum variation, as defined in text. b) (Middle panel) The same as a) but for $\mathrm{CO}(1-0)$. c) (Lower panel) The same as a) but for $\mathrm{CO}(2-1)$. Maps are deprojected onto the galaxy plane.

Fig. 16c: $Q_{2}>Q_{1}$, except for the inner $r \sim 50$ pc, i.e., below the limit of the spatial resolution of the image ${ }^{3}$. This has

\footnotetext{
3 The peak of $Q_{1}$ at $r \sim 50-100$ pc identified in Fig. 16a is an artifact created by the spatial smoothing performed to derive the large-scale representation of $\Phi(R, \theta)$, and as such is unreliable.
} 
consequences on the interpretation of the lopsidedness identified in the gas distribution and kinematics probed by $\mathrm{CO}$. Lopsidedness cannot be clearly attributed to the action of a true $m=1$ mode in the stellar potential. As a second important difference, the oval identified in the $K$-band image was not present in the $I$-band image of GB05. The reported discrepancies in $\Phi(R, \theta)$ can be attributed to the residual effects of extinction which are noticeable in the central $r \sim 2 \mathrm{kpc}$ of the $I$-band image.

Taken together, these differences are relevant to explaining the new version of the gravity torque budget in NGC 4579, described in Sect. 8.2.2.

\subsubsection{Gas-flow timescales in NGC 4579}

Figure 18 shows the 2D pattern of gravitational torques obtained throughout the disk of NGC 4579, from $r \sim 15 \mathrm{kpc}$ down to the inner $r \sim 50 \mathrm{pc}$ around the AGN. The derived torques change sign following a characteristic 2D butterfly pattern. Quadrants I-to-IV will define hereafter the regions where the signs of the torques driven by the dominant perturbation of the stellar potential are expected to be constant.

Assuming that the gas is rotating counterclockwise, Fig. 18 shows that, although HI emission is distributed along the four quadrants, the majority of the gas is seen to lie along the leading edges of the large-scale bar where torques are negative (quadrants II( $(-)$ and IV $(-)$ ), especially on intermediate scales (4 kpc $<$ $r<8 \mathrm{kpc})$. Figure 19a shows that the azimuthally averaged torques are systematically negative from $r \sim 10 \mathrm{kpc}$ down to $r \sim$ $4 \mathrm{kpc}$. This result suggests that the corotation barrier of the bar in NGC 4579 (at $\sim 6 \mathrm{kpc}$ ) has been overcome due to secular evolution processes. If the gas response was the canonical spiral pattern coupled to the bar, the corotation would separate in the disk the regions where torques are positive (from corotation to the outer Lindblad resonance (OLR)) from those where torques are negative (from corotation to the oILR). Positive torques would tend to form a ring at the OLR. However, a decoupling of the spiral allows the gas to efficiently populate the UHR region inside corotation and thus produce net gas inflow on intermediate scales in the disk. A similar scenario has been suggested to explain the gravity torque maps of some of the NUGA galaxies recently analyzed by Haan et al. (2008b). The implied timescales for angular momentum transfer in NGC 4579 are $\sim 5-10$ rotation periods. On its way to the nucleus, the gas stops in the outer disk close to the UHR of the bar, where torques become negligible (Fig. 19a).

In the inner disk, Fig. 18 shows that the bulk of the $\mathrm{CO}$ emission along the inner spiral arms lies where torques are strong and negative (quadrants II $(-)$ and IV $(-)$ ). The corresponding azimuthally averaged torques, shown in Fig. 19, are mostly negative from $r \sim 1.3 \mathrm{kpc}$ down to $r \sim 300 \mathrm{pc}$. This indicates a net gas inflow from the oILR down to a region inside the iILR of the bar. As for the outer disk, the implied timescales for angular momentum transfer are also $\sim 5-10$ rotation periods. This result is in agreement with our first calculation published by GB05 (see Fig. 11 of GB05) regarding the sign of the torques. However the gravity torques shown in Figs. 19b and c are comparatively stronger at the same radii, in particular those contributing to gas inflow. The changing picture can be attributed to the new $\Phi(R, \theta)$ used in this work but, also, to the new $N(x, y)$-values that now include the short spacing correction for CO. This is illustrated in Fig. 20 where we compare the $\mathrm{d} L / L$ curves obtained with the new $\Phi(R, \theta)$ used in this work, but with two different versions of $N(x, y)$, derived from the PdBI-only CO data (used by GB05), and from the short spacing corrected PdBI+30 $\mathrm{m}$ data
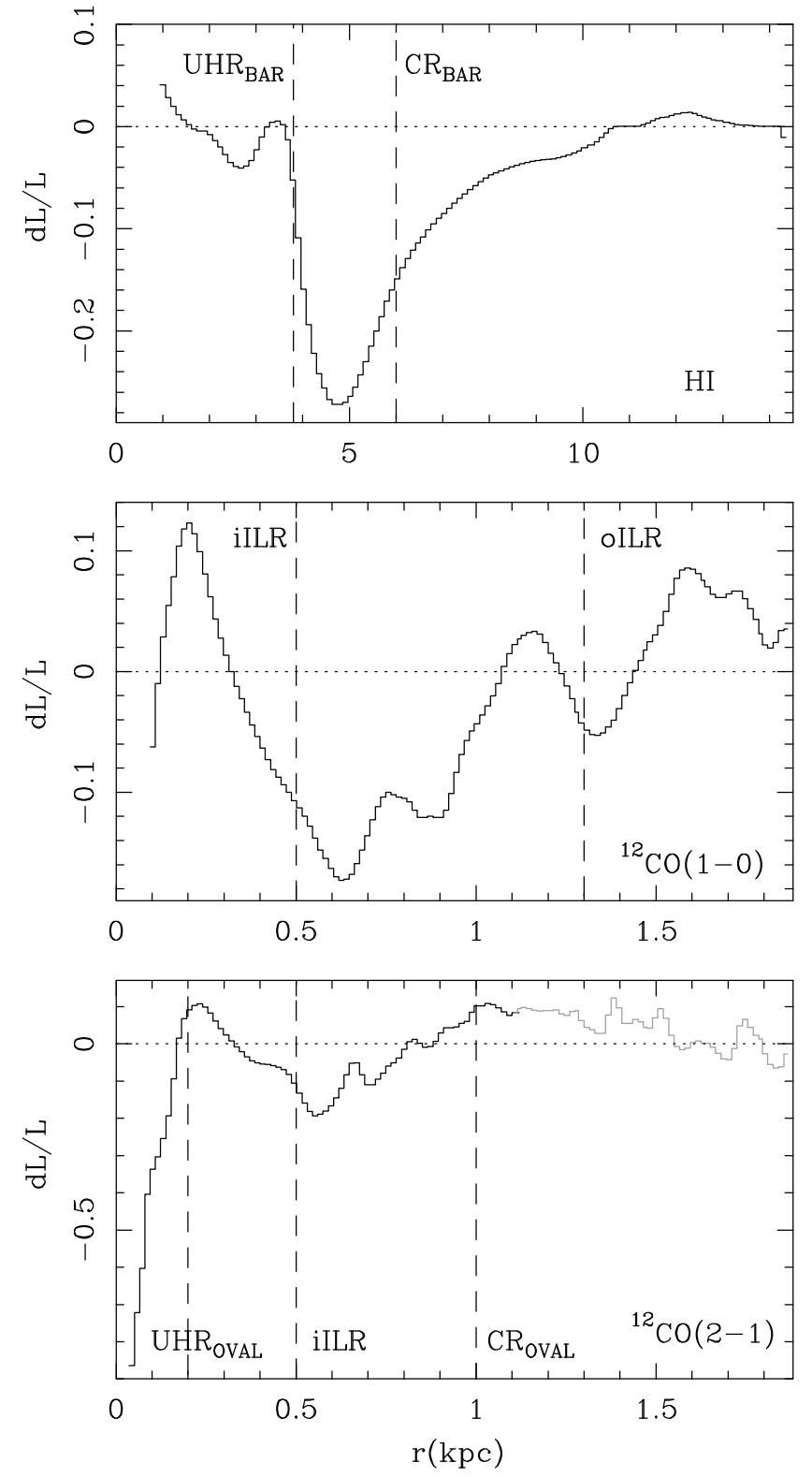

Fig. 19. The average fraction of the angular momentum transferred from/to the gas in one rotation $-\mathrm{d} L / L-$ are plotted as a function of radius, as derived from the $\mathrm{HI}$ (upper panel), $\mathrm{CO}(1-0)$ (middle panel) and $\mathrm{CO}(2-1)$ (lower panel) maps of the disk of NGC 4579. The locations of BAR resonances (iILR, oILR, $\mathrm{CR}_{\mathrm{BAR}}$, $\mathrm{UHR}_{\mathrm{BAR}}$ ) and those of OVAL resonances $\left(\mathrm{CR}_{\mathrm{OVAL}}, \mathrm{UHR}_{\mathrm{OVAL}}\right)$ are highlighted. We have blocked the central $r \sim 1 \mathrm{kpc}$ in the $\mathrm{d} L / L$ plot obtained from the HI map, due to the spatial resolution limit of the HI data. For similar reasons, the central $r \sim 100 \mathrm{pc}$ and $r \sim 50 \mathrm{pc}$ around the AGN are blocked in the $\mathrm{d} L / L$ plots obtained from the CO $1-0$ and 2-1 maps, respectively. In the range $1.8 \mathrm{kpc}>r>1.2 \mathrm{kpc}$, estimates of $\mathrm{d} L / L$ based on the $\mathrm{CO}(1-0)$ map are more reliable than those derived from $\mathrm{CO}(2-1)$ data (in grey), as the latter come from a region outside the PdBI primary beam at this frequency. The same display conventions are applied to Figs. 21 to 23.

(this work), respectively. In summary, we find that the gas in the inner disk is funneled from the oILR to a region inside the iILR at $r \sim 300 \mathrm{pc}$. There is a barrier of positive torques between $r \sim$ $200 \mathrm{pc}$ (i.e., close to the UHR of the oval perturbation) and $r \sim$ $300 \mathrm{pc}$ (Figs. 19b and c).

Closer to the AGN $(r<200 \mathrm{pc})$, the bulk of the CO emission is concentrated north of the nucleus (complex N) in the trailing 

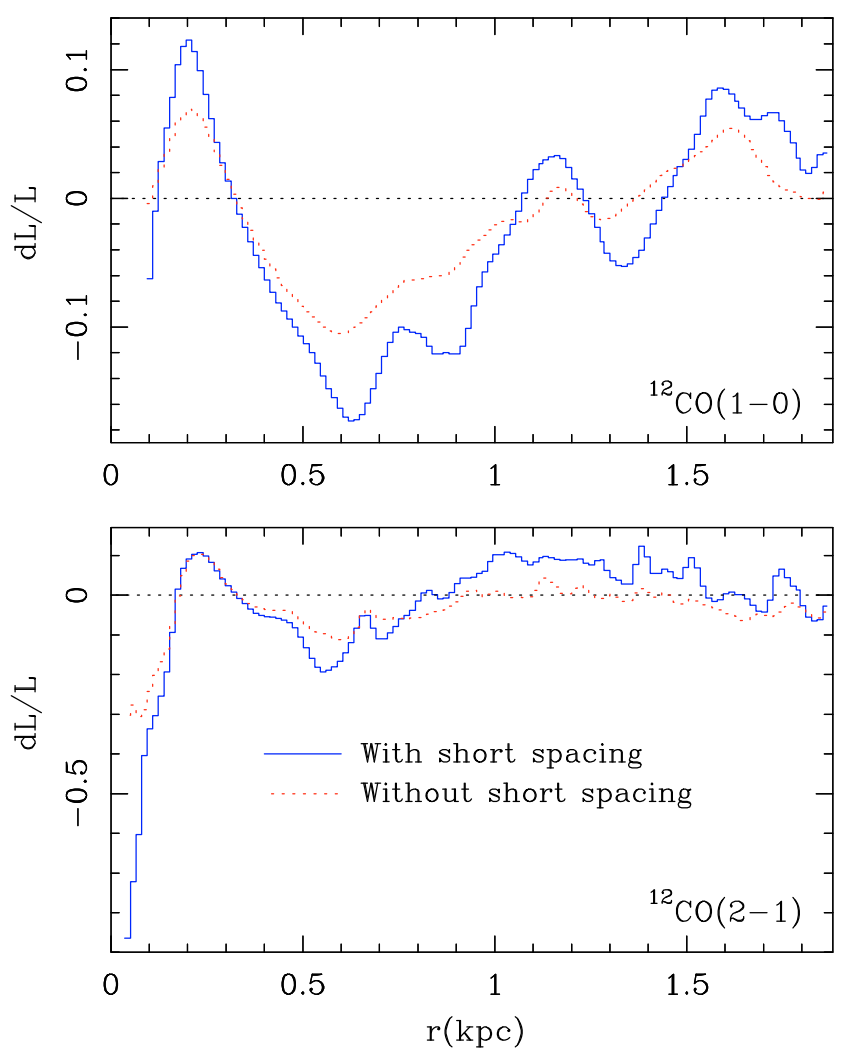

Fig. 20. Same as Fig. 19, but here showing the results obtained for $\mathrm{d} L / L$ from CO maps with (continuous line) and without (dashed line) short spacing correction.

quadrant of the large-scale bar $\mathrm{I}(+)$, where stellar torques are positive. However, the rest of the central disk complexes from $r \sim 50 \mathrm{pc}$ to $r \sim 150 \mathrm{pc}$, identified in the $\mathrm{CO}(2-1)$ map, feel negative torques due to the combined action of the large-scale bar and the inner oval. The two $m=2$ modes act in concert to produce net gas inflow down to the spatial resolution of our observations (see Fig. 19c). This result is different to that reported in GB05, where the evidence of AGN fueling at $r<200 \mathrm{pc}$ was lacking. The implied timescales for angular momentum transfer are extremely short: $\sim 1-3$ rotation periods.

\subsubsection{Mass transfer rates in NGC 4579}

In terms of mass inflow/outflow rates, the feeding budget integrated out to a certain radius is clearly negative in the outer disk of NGC 4579 ( $r>4 \mathrm{kpc})$. As shown in Fig. 21, $\mathrm{d} M / \mathrm{d} t$ goes from virtually zero inflow at $r \sim 4 \mathrm{kpc}$ to $\sim-0.3 M_{\odot} \mathrm{yr}^{-1}$ at $r \sim 10 \mathrm{kpc}$. The minimum of $d^{2} M / \mathrm{d} R \mathrm{~d} t$, indicative of the largest inflow rate per unit length, is reached at $r \sim 5.5 \mathrm{kpc}$, a position close to the corotation of the bar. As argued in Sect. 8.2.2, this paradoxical result can be explained if the gas response in the outer disk is decoupled from the bar potential. The inflow rate integrated on these scales $\left(\mathrm{d} M / \mathrm{d} t[4 \mathrm{kpc}<r<10 \mathrm{kpc}] \sim-0.3 M_{\odot} \mathrm{yr}^{-1}\right)$ is a factor $\sim 4$ lower than the gas feeding needs implied by the current SFR integrated on this region $\left(S F R_{\text {outer }} \sim 1.3 M_{\odot} \mathrm{yr}^{-1}\right.$, as derived in Sect. 7.4). Note, however, that on these scales, the $\mathrm{d} M / \mathrm{d} t$ estimated from $\mathrm{HI}$ is a lower limit to the total inflow rate, as it does not include the contribution from molecular gas at these radii.

In the inner disk, the integrated mass feeding budget is systematically negative from $r \sim 2 \mathrm{kpc}$ down to $r \sim 400 \mathrm{pc}$. As shown in Figs. 22 and 23, $\mathrm{d} M / \mathrm{d} t$ goes from $\sim-0.5 M_{\odot} \mathrm{yr}^{-1}$ at

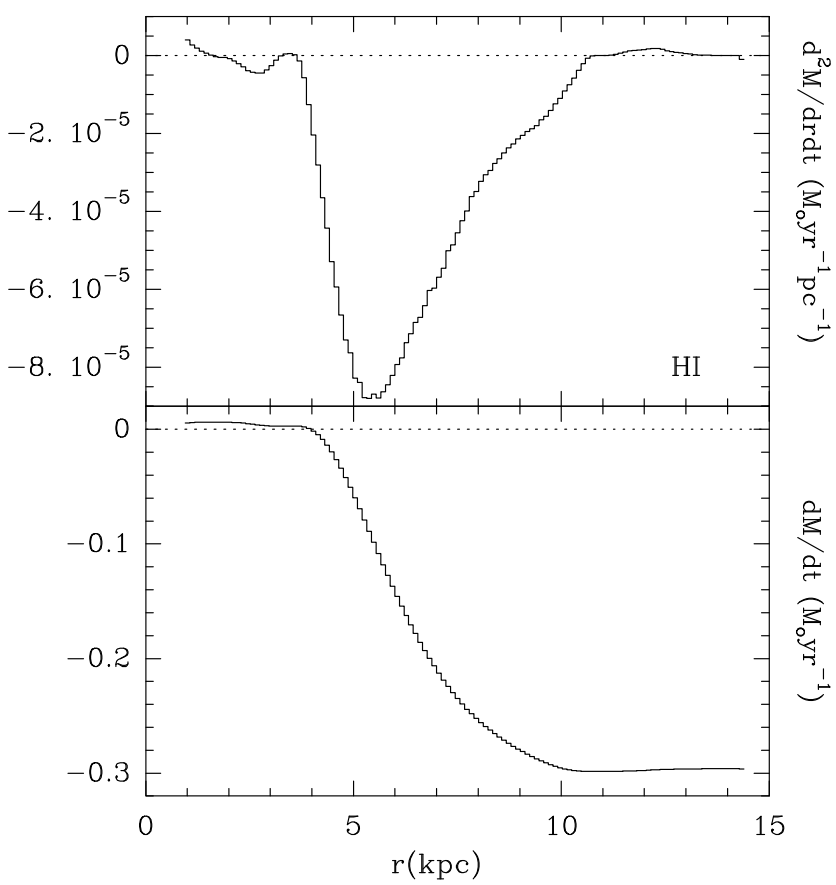

Fig. 21. a) (Upper panel) We represent the radial variation of the mass inflow(-) or outflow (+) rate of gas per unit radial length $\left(\mathrm{d}^{2} M / \mathrm{d} R \mathrm{~d} t\right)$ in the nucleus of NGC 4579 due to the action of stellar gravitational torques on the gas distribution as derived from HI data. Units are $M_{\odot} \mathrm{yr}^{-1} \mathrm{pc}^{-1}$. b) (Lower panel) We plot the mass inflow/outflow rate integrated inside a certain radius $r(\mathrm{~d} M / \mathrm{d} t)$ in $M_{\odot} \mathrm{yr}^{-1}$.

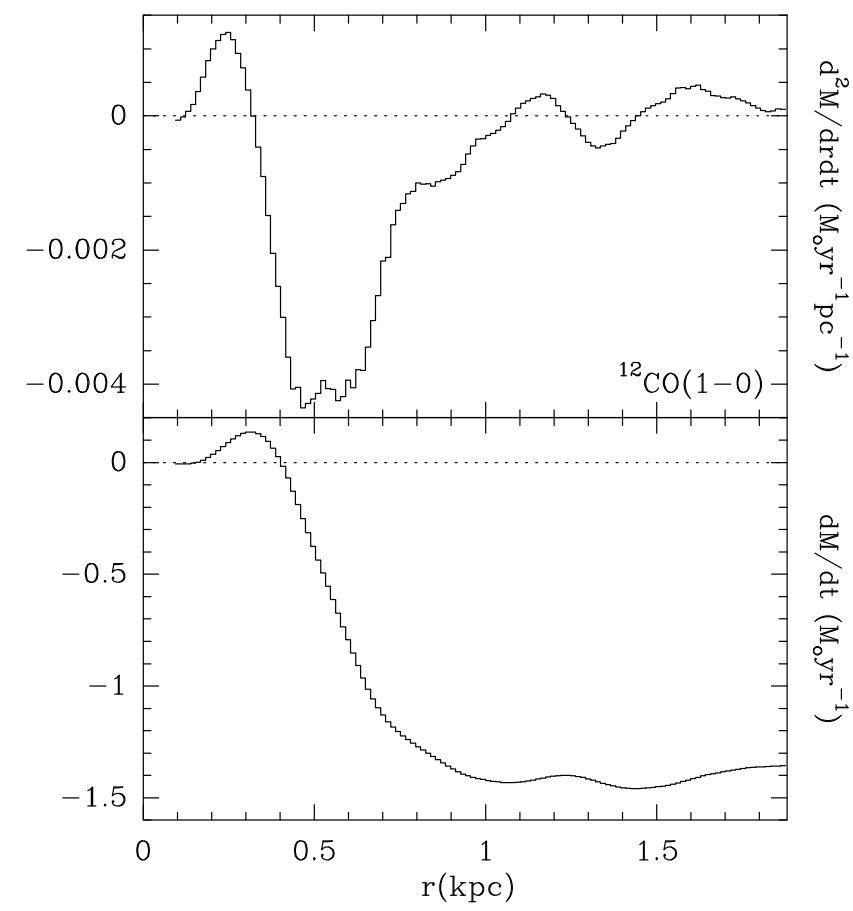

Fig. 22. Same as Fig. 21 but here based on the gas distribution as derived from $\mathrm{CO}(1-0)$ data.

the iILR (at $500 \mathrm{pc}$ ) to $\sim-1.5 M_{\odot} \mathrm{yr}^{-1}$ at the oILR (at $1.3 \mathrm{kpc}$ ). This is a factor $\sim 5$ larger than the corresponding $\mathrm{d} M / \mathrm{d} t$ inflow rates measured for the outer disk $(r>4 \mathrm{kpc})$. The minimum of $\mathrm{d}^{2} M / \mathrm{d} R \mathrm{~d} t$, indicative of the largest inflow rate per unit length, is reached in the vicinity of the iILR. The inflow rate integrated on these scales $\left(\mathrm{d} M / \mathrm{d} t[400 \mathrm{pc}<r<2 \mathrm{kpc}] \sim-1.5 M_{\odot} \mathrm{yr}^{-1}\right)$ 


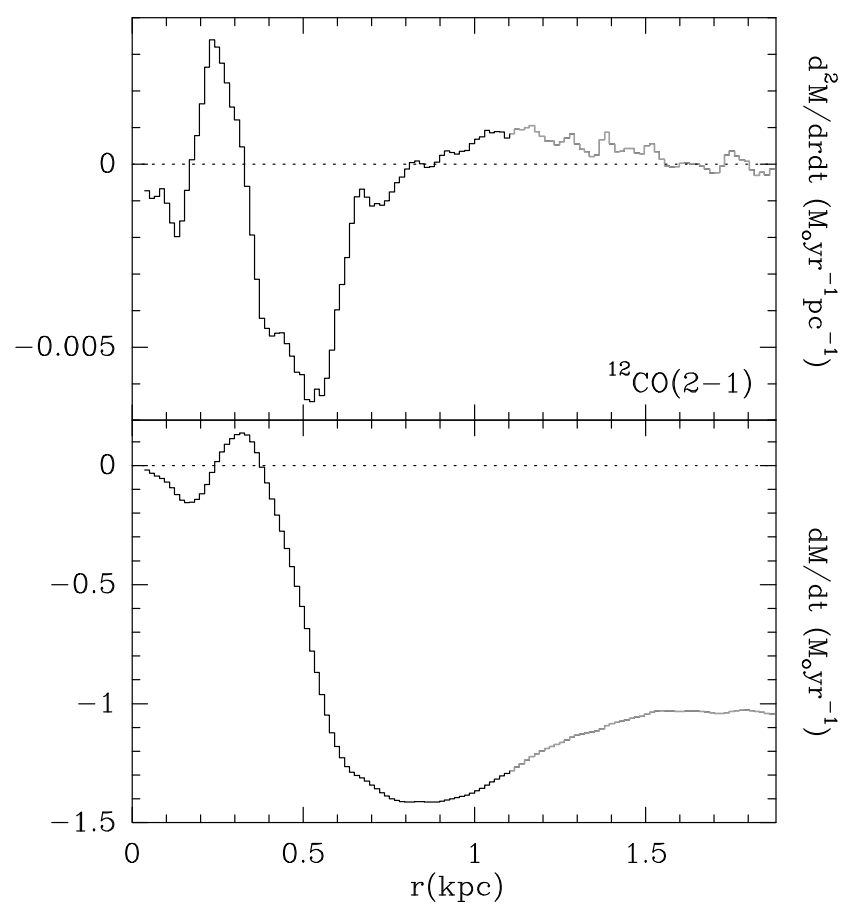

Fig. 23. Same as Fig. 21 but here based on the gas distribution as derived from $\mathrm{CO}(2-1)$ data.

is an order of magnitude higher than the gas feeding rate required to sustain the current SFR in this region $\left(S F R_{\text {inner }} \sim\right.$ $0.10 M_{\odot} \mathrm{yr}^{-1}$, as derived in Sect. 7.4). The timescale for gas consumption by star formation is significantly larger than the dynamical timescale for inflow in the inner disk at present.

Closer to the AGN $(r<200 \mathrm{pc})$, the integrated mass budget derived from the $\mathrm{CO}(2-1)$ map shows negative torques down to $r \sim 50 \mathrm{pc}$. The minimum of $\mathrm{d}^{2} M / \mathrm{d} R \mathrm{~d} t$, indicative of the largest inflow rate per unit length, is reached inside the UHR of the oval, at $r \sim 100 \mathrm{pc}$. The integrated inflow rate measured at $r \sim 50 \mathrm{pc}$, the position closest to the AGN where $\mathrm{d} M / \mathrm{d} t$ can be estimated down to the limit imposed by the spatial resolution of our observations, is $\mathrm{d} M / \mathrm{d} t \sim-8 \times 10^{-3} M_{\odot} \mathrm{yr}^{-1}$. This inflow rate can satisfactorily explain the AGN activity of NGC 4579: the inferred $\mathrm{d} M / \mathrm{d} t$ lies within the range of the mass accretion rates derived from the typical bolometric luminosities in low-luminosity AGNs $\left(\sim 10^{-2}\right.$ to $\sim 10^{-5} M_{\odot} \mathrm{yr}^{-1}$ from Seyfert to LINERs; see compilation by Jogee 2006).

\section{Summary and conclusions}

In this paper we have studied the efficiency of angular momentum transport in the LINER/Seyfert 1.9 spiral NGC 4579 using a complete gravity torque map of the disk of the galaxy. Gas flow rates from the outer disk region at $r \sim 15 \mathrm{kpc}$ down to the inner disk at $\sim$ a few $10 \mathrm{pc}$ are derived to search for signatures of secular evolution in the fueling process. The CO maps obtained with the PdBI and the $30 \mathrm{~m}$ telescope as part of the NUGA survey are used to derive the gravity torque budget in the circumnuclear regions. Gravity torques on the outer disk gas are derived using the HI map obtained with the VLA. The stellar potential is derived from a $K$-band wide field image of the galaxy. We summarize the main results of our study as follows:

- By analyzing the distribution and kinematics of the gas and their relation to other tracers of the ISM and the stellar structure we identify signs of dynamical decoupling of several patterns on different spatial scales of the disk. The $K$-band image reveals a stellar bar of diameter $D \sim 12 \mathrm{kpc}$ and moderate strength, as well as a nested weak oval distortion in the inner $r \sim 150 \mathrm{pc}$ of the disk. The bar and the oval are not aligned, a signature of dynamical decoupling. Molecular gas in the inner $r \leq 2 \mathrm{kpc}$ disk is distributed in two spiral arms, an outer arc, and a central lopsided disk-like structure. The morphology of the outer disk revealed by HI shows that the neutral gas is currently piling up in a pseudo-ring at $r \geq 4 \mathrm{kpc}$. The pseudo-ring is formed by two winding spiral arms that are morphologically decoupled from the bar structure; this suggests that the spirals and the bar do not share a common pattern speed.

- We have estimated the pattern speeds and principal resonances of the bar and the oval in the disk of the galaxy. In the scenario of dynamical decoupling, the oval has a higher pattern speed compared to the primary bar. The gas response in the circumnuclear regions traced by the $\mathrm{CO}$ maps follows the expected gas flow pattern induced by the bar potential in the presence of two ILRs at $r \sim 500 \mathrm{pc}$ (iILR) and $r \sim$ $1.3 \mathrm{kpc}$ (oILR). We can explain the decoupling of the oval with respect to the large-scale bar, as the corotation of the oval would be close to the oILR of the large-scale bar. The pseudo-ring detected in $\mathrm{HI}$ is located inside the bar corotation $\left(r_{\mathrm{CR}} \sim 6 \mathrm{kpc}\right)$ and close to the predicted position of the UHR of the bar $\left(r_{\mathrm{UHR}} \sim 3.8 \mathrm{kpc}\right)$.

- The derived gravity torque budget in NGC 4579 shows that the fueling process is at work on different spatial scales in the disk. In the outer disk, the decoupling of the spiral allows the gas to efficiently populate the UHR region inside corotation and thus produce net gas inflow on intermediate scales. This suggests that the corotation barrier of the bar has been overcome due to secular evolution processes. The gas stops close to the UHR of the bar, where torques become negligible. The gas in the inner disk is efficiently funneled by gravity torques from the oILR down to $r \sim 300 \mathrm{pc}$. Closer to the AGN ( $r<200 \mathrm{pc}$ ), gas feels negative torques due to the combined action of the large-scale bar and the inner oval. The two $m=2$ modes act in concert to produce net gas inflow down to the spatial resolution of our observations, providing a clear smoking gun evidence of fueling.

- In the inner disk, the mass feeding budget is systematically negative from $r \sim 2 \mathrm{kpc}$ down to $r \sim 400 \mathrm{pc}$. The inflow rate integrated on these scales $\left(\sim-1.5 M_{\odot} \mathrm{yr}^{-1}\right)$ is an order of magnitude higher than the gas feeding rate required to sustain the current SFR in this region. Closer to the AGN $(r<200 \mathrm{pc})$, the torques are negative down to $r \sim 50 \mathrm{pc}$. The integrated inflow rate measured at $r \sim 50 \mathrm{pc}$ is $\sim-8 \times$ $10^{-3} M_{\odot} \mathrm{yr}^{-1}$. This inflow rate is consistent with the requirements of the AGN activity of NGC 4579.

NGC 4579 is a representative example of a LLAGN in which a hierarchy of mechanisms can act in concert to efficiently transport the gas from the outer disk (on kpc scales) down to vicinity of the AGN (on a few 10 pc scales) through the action of gravity torques. The decoupling of dynamical modes on several spatial scales (outer spiral, bar, oval) makes it possible to drain the angular momentum of the gas and feed the star formation in the disk, and possibly the AGN activity itself. In the last step down to the AGN, viscosity can also play a significant role in the fueling process (e.g., see discussion in GB05). In particular, we estimate that the efficiency of viscosity at a typical distance of $r \sim 50 \mathrm{pc}$ around the central engine of NGC $4579^{4}$ may be

${ }^{4}$ Originally estimated by GB05 at $r \sim 200$ pc. 
comparable to the efficiency of gravity torques. Both mechanisms can combine their efforts and produce sufficient AGN feeding on extremely short dynamical timescales of $\sim 1-2$ rotation periods.

Acknowledgements. We acknowledge the IRAM staff from the Plateau de Bure and from Grenoble for carrying out the observations and help provided during the data reduction. We thank Roberto Neri for fruitful discussions. I.M. acknowledges financial support from the Spanish Ministerio de Ciencia y Tecnología (grant AYA2007-62190) and from the Junta de Andalucía (grant TIC-144).

\section{References}

Anderson, J. M., Ulvestad, J. S., \& Ho, L. C. 2004, ApJ, 603, 42

Athanassoula, E. 1992, MNRAS, 259, 345

Baker, A. J. 2000, Ph.D. Thesis

Baker, A. J., Jogee, S., Sakamoto, K., \& Scoville, N. Z. 2003, Active Galactic Nuclei: From Central Engine to Host Galaxy, 290, 479

Barth, A. J., Reichert, G. A., Filippenko, A. V., et al. 1996, AJ, 112, 1829

Barth, A. J., Ho, L. C., Filippenko, A. V., Rix, H.-W., \& Sargent, W. L. W. 2001, ApJ, 546, 205

Bohlin, R. C., Savage, B. D., \& Drake, J. F. 1978, ApJ, 224, 132

Boone, F., Baker, A. J., Schinnerer, E., et al. 2007, A\&A, 471, 113

Braine, J., \& Combes, F. 1992, A\&A, 264, 433

Buta, R., \& Combes, F. 1996, Fund. Cosmic Phys., 17, 95

Canzian, B. 1993, ApJ, 414, 487

Cardelli, J. A., Clayton, G. C., \& Mathis, J. S. 1989, ApJ, 345, 245

Casasola, V., Combes, F., García-Burillo, S., et al. 2008, A\&A, 490, 61

Chemin, L., Balkowski, C., Cayatte, V., et al. 2006, MNRAS, 366, 812

Combes, F. 2003, in Active Galactic Nuclei: from Central Engine to Host Galaxy, ed. S. Collin, F. Combes, \& I. Shlosman, ASP Conf. Ser., 290, 411

Combes, F. 2008, IAU Symp., 245, 151

Combes, F., \& Sanders, R. H. 1981, A\&A, 96, 164

Combes, F., García-Burillo, S., Boone, F., et al. 2004, A\&A, 414, 857

Comerón, S., Knapen, J. H., Beckman, J. E., \& Shlosman, I. 2008, A\&A, 478, 403

Contini, M. 2004, MNRAS, 354, 675

Daigle, O., Carignan, C., Amram, P., et al. 2006, MNRAS, 367, 469

Dewangan, G. C., Griffiths, R. E., Di Matteo, T., \& Schurch, N. J. 2004, ApJ, 607,788

Elfhag, T., Booth, R. S., Hoeglund, B., Johansson, L. E. B., \& Sandqvist, A. 1996, A\&AS, 115, 439

Eracleous, M., Shields, J. C., Chartas, G., \& Moran, E. C. 2002, ApJ, 565, 108

Eskridge, P. B., Frogel, J. A., Pogge, R. W., et al. 2002, ApJS, 143, 73

Ferrarese, L., \& Ford, H. 2005, Space Sci. Rev., 116, 523

Filippenko, A. V., \& Sargent, W. L. W. 1985, ApJS, 57, 503

García-Burillo, S., Guelin, M., \& Cernicharo, J. 1993, A\&A, 274, 123

García-Burillo, S., Sempere, M. J., Combes, F., \& Neri, R. 1998, A\&A, 333, 864

García-Burillo, S., Combes, F., Eckart, A., et al. 2003a, in Active Galactic Nuclei: from Central Engine to Host Galaxy, ed. S. Collin, F. Combes, \& I. Shlosman, ASP Conf. Ser., 423

García-Burillo, S., Combes, F., Hunt, L. K., et al. 2003b, A\&A, 407, 485

García-Burillo, S., Combes, F., Schinnerer, E., Boone, F., \& Hunt, L. K. 2005, A\&A, 441, 1011

Gil de Paz, A., Boissier, S., Madore, B. F., et al. 2007, ApJS, 173, 185

Guilloteau, S., \& Lucas, R. 2000, in Imaging at Radio through Submillimeter Wavelengths, ed. J. G. Mangum, \& S. J. E. Radford, ASP Conf. Ser., 299

Guhathakurta, P., van Gorkom, J. H., Kotanyi, C. G., \& Balkowski, C. 1988, AJ, 96, 851

Haan, S., Schinnerer, E., Mundell, C. G., García-Burillo, S., \& Combes, F. 2008a, AJ, 135, 232

Haan, S., Schinnerer, E., Emsellem, E., et al. 2008b [arXiv: 0811. 1988]
Héraudeau, P., \& Simien, F. 1998, A\&AS, 133, 317

Ho, L. C., \& Ulvestad, J. S. 2001, ApJS, 133, 77

Ho, L. C., Filippenko, A. V., \& Sargent, W. L. W. 1997, ApJS, 112, 315

Ho, L. C., Feigelson, E. D., Townsley, L. K., et al. 2001, ApJ, 549, L51

Holtzman, J. A., Burrows, C. J., Casertano, S., et al. 1995, PASP, 107, 1065

Hopkins, P. F., \& Hernquist, L. 2006, ApJS, 166, 1

Huchra, J. P., Wyatt, W. F., \& Davis, M. 1982, AJ, 87, 1628

Hummel, E., van der Hulst, J. M., Keel, W. C., \& Kennicutt, R. C., Jr. 1987, A\&AS, 70, 517

Hunt, L. K., Combes, F., García-Burillo, S., et al. 2008, A\&A, 482, 133

Hutchings, J. B., \& Neff, S. G. 1992, AJ, 104, 1

Jarrett, T. H., Chester, T., Cutri, R., Schneider, S. E., \& Huchra, J. P. 2003, AJ, 125,525

Jogee, S. 2006, Physics of Active Galactic Nuclei at all Scales, 693, 143

Jogee, S., Baker, A. J., Sakamoto, K., et al. 2001, in The Central Kiloparsec of Starbursts and AGN: The La Palma Connection, ASP Conf. Ser., 249, 612

Kenney, J. D., \& Young, J. S. 1986, ApJ, 301, L13

Kenney, J. D., \& Young, J. S. 1988, ApJS, 66, 261

Kenney, J. D. P., \& Young, J. S. 1989, ApJ, 344, 171

Kennicutt, R. C., Jr. 1998, ARA\&A, 36, 189

Kennicutt, R. C., Jr., Armus, L., Bendo, G., et al. 2003, PASP, 115, 928

King, A. R., \& Pringle, J. E. 2007, MNRAS, 377, L25

Knapen, J. H., Shlosman, I., \& Peletier, R. F. 2000, ApJ, 529, 93

Knapen, J. H., de Jong, R. S., Stedman, S., \& Bramich, D. M. 2003, MNRAS, 344,527

Kohno, K., Matsushita, S., Vila-Vilaró, B., et al. 2001 in The Central Kiloparsec of Starbursts and AGN: The La Palma Connection, ASP Conf. Ser., 249, 672

Koopmann, R. A., Kenney, J. D. P., \& Young, J. 2001, ApJS, 135, 125

Krause, M., Fendt, C., \& Neininger, N. 2007, A\&A, 467, 1037

Krips, M., Eckart, A., Neri, R., et al. 2005, A\&A, 442, 479

Krips, M., Eckart, A., Krichbaum, T. P., et al. 2007, A\&A, 464, 553

Kuno, N., Sato, N., Nakanishi, H., et al. 2007, PASJ, 59, 117

Lindt-Krieg, E., Eckart, A., Neri, R., et al. 2008, A\&A, 479, 377

Maoz, D., Koratkar, A., Shields, J. C., et al. 1998, AJ, 116, 55

Maoz, D., Nagar, N. M., Falcke, H., \& Wilson, A. S. 2005, ApJ, 625, 699

Márquez, I., Durret, F., Masegosa, J., et al. 2000, A\&A, 360, 431

Martini, P. 2004, in Coevolution of Black Holes and Galaxies, ed. L. C. Ho (Cambridge University Press), 170

Mulchaey, J. S., \& Regan, M. W. 1997, ApJ, 482, L135

Pahre, M. A., Ashby, M. L. N., Fazio, G. G., \& Willner, S. P. 2004, ApJS, 154, 235

Palacios, J., Garcia-Vargas, M. L., Diaz, A., Terlevich, R., \& Terlevich, E. 1997, A\&A, 323, 749

Paturel, G., Fang, Y., Petit, C., Garnier, R., \& Rousseau, J. 2000, A\&AS, 146, 19

Pogge, R. W. 1989, ApJS, 71, 433

Pogge, R. W., Maoz, D., Ho, L. C., \& Eracleous, M. 2000, ApJ, 532, 323

Regan, M. W., \& Teuben, P. 2003, ApJ, 582, 723

Rubin, V. C., Waterman, A. H., \& Kenney, J. D. P. 1999, AJ, 118, 236

Sellwood, J. A., \& Sparke, L. S. 1988, MNRAS, 231, 25P

Sanders, D. B., Mazzarella, J. M., Kim, D.-C., Surace, J. A., \& Soifer, B. T. 2003, AJ, 126, 1607

Solomon, P. M., \& Barrett, J. W. 1991, in Dynamics of Galaxies and their Molecular Cloud Distributions, ed. F. Combes, \& F. Casoli (Kluwer Academic Publishers), from the IAU Symp., 146, 235

Stauffer, J. R. 1982, ApJ, 262, 66

Terashima, Y., Ho, L. C., Ptak, A. F., et al. 2000, ApJ, 535, L79

Tully, R. B., \& Fisher, J. R. 1988, Catalog of Nearby Galaxies, ed. R. B. Tully, \& J. R. Fisher (Cambridge, UK: Cambridge University Press), 224

Ulvestad, J. S., \& Ho, L. C. 2001, ApJ, 562, L133

Vollmer, B., Braine, J., Balkowski, C., Cayatte, V., \& Duschl, W. J. 2001, A\&A, 374,824

Wada, K. 2004, in Coevolution of Black Holes and Galaxies, ed. L. C. Ho (Cambridge University Press), 187

Young, C. K., \& Currie, M. J. 1998, A\&AS, 127, 367 